\title{
HOW REACTION-DIFFUSION PDES APPROXIMATE THE LARGE-POPULATION LIMIT OF STOCHASTIC PARTICLE MODELS.
}

\author{
S. ISAACSON, J. MA AND K. SPILIOPOULOS
}

\begin{abstract}
Reaction-diffusion PDEs and particle-based stochastic reaction-diffusion (PBSRD) models are commonly-used approaches for modeling the spatial dynamics of chemical and biological systems. Standard reaction-diffusion PDE models ignore the underlying stochasticity of spatial transport and reactions, and are often described as appropriate in regimes where there are large numbers of particles in a system. Recent studies have proven the rigorous large-population limit of PBSRD models, showing the resulting mean-field models (MFM) correspond to non-local systems of partial-integro differential equations. In this work we explore the rigorous relationship between standard reaction-diffusion PDE models and the derived MFM. We prove that the former can be interpreted as an asymptotic approximation to the later in the limit that bimolecular reaction kernels are short-range and averaging. As the reactive interaction length scale approaches zero, we prove the MFMs converge at second order to standard reaction-diffusion PDE models. In proving this result we also establish local well-posedness of the MFM model in time for general systems, and global well-posedness for specific reaction systems and kernels. Finally, we illustrate the agreement and disagreement between the MFM, SM and the underlying particle model for several numerical examples.
\end{abstract}

\section{INTRODUCTION}

Reaction-diffusion partial differential equations (PDEs) are often used to model the average or largepopulation dynamics of systems of reacting and diffusing particles at a macroscopic level. In biological contexts, such models may describe signaling pathways inside cells, interactions within populations (of cells, animals or people), or chemical signals within tissues. Standard mass-action-based reaction-diffusion PDE models (subsequently SM for standard model) in the form of Eq. (2.2) in Section 2, are extensively used in modeling studies, for example [ECM07, MNKS09, NTS08]. They can be formally obtained from classical mass-action ODE models for chemical reactions [G00] by adding a diffusion operator (or more generally a second order elliptic operator) to model spatial transport. However, such a formulation does not capture the detailed spatial reaction mechanisms described in more microscopic particle-based stochastic reactiondiffusion (PBSRD) models [TS67, D76a, D76b]. The latter explicitly resolve the diffusion of, and reactions between, individual molecules, which are often represented as point particles.

Due to their mathematical complexity and high dimensionality, PBSRD models are typically studied by Monte Carlo simulations approximating the underlying stochastic process of molecules diffusing and reacting. This is often computationally expensive, particularly in systems with large populations for which the dynamics are well-approximated as deterministic. In order to deal with this issue, coarse-grained models were developed in [LLN19, IMS20] by proving the large population mean-field limit of the measure-valued stochastic process (MVSP) tracing the empirical distribution of the particles. In this limit the empirical distribution can be rigorously shown to satisfy an evolution equation for which the corresponding limiting density satisfies a system of partial-integro differential equations (PIDEs), e.q. Eq. (2.1) in Section 2. We subsequently call these PIDEs the mean-field model (MFM). In contrast to the SM, the nonlocal MFM retains the detailed spatial reaction mechanisms of the underlying PBSRD model.

The new contributions of this paper are to study the relationship between the formal SM and the coarsegrained and rigorously derived MFM for PBSRD systems. We show that the SM can be interpreted as an asymptotic approximation to the more general MFM when the underlying reactive interaction kernels are averaging and short-range. In particular, we prove that the solution to the MFM converges to the

\footnotetext{
${ }^{1}$ Boston University, Department of Mathematics and Statistics, 111 Cummington Mall, Boston, 02215. E-mails: isaacson@math.bu.edu,majw@bu.edu, kspiliop@math.bu.edu

Date: June 2, 2021.

K.S. was partially supported by the NSF DMS-1550918, Simons Foundation Award 672441 and ARO W911NF-20-1-0244. J. M. and S. A. I. were partially supported by NSF DMS-1902854 and ARO W911NF-20-1-0244.
} 
solution of the SM for such kernels as the kernel's width approaches zero. The convergence is proven to be second order in the approximation parameter that captures the kernel width, see Theorem 3.1. We also demonstrate by numerical simulations the convergence as the kernel width approaches zero of the MFM to the SM, show how well the MFM and SM agree with the average concentration fields of PBSRD models, and demonstrate that when the reactive interaction distance between two particles is not sufficiently "small" the SM may provide a poor approximation to either the MFM or mean concentration fields of the PBSRD models. To illustrate a biologically-relevant case where the MFM is needed, we conclude with a simple model motivated by our recent study of T-cell receptor signaling [ZI20]. There it is not immediately clear how to even define an appropriate SM. In contrast, we demonstrate that the (well-defined) MFM correctly reproduces the qualitative steady-state behavior of the underlying particle model observed in [ZI20].

As our main result is presented for systems with general reaction networks, and relatively notationally heavy, we now give a brief summary of it in the special case of the multi-particle $\mathrm{A}+\mathrm{B} \leftrightarrows \mathrm{C}$ reaction. We assume all molecules move by Brownian motion in $\mathbb{R}^{d}$ with species-dependent diffusivities, $D^{\mathrm{A}}, D^{\mathrm{B}}$ and $D^{\mathrm{C}}$. Assume an A molecule at $x$ and B molecule at $y$ may react with probability per time $\hat{K}_{1}^{\epsilon}(x-y) / \gamma$. Here $\gamma$ denotes a large system size parameter, for example Avogadro's number in $\mathbb{R}^{d}$ [LLN19, IMS20]. $\epsilon$ denotes the length scale of the kernel, related to our assumption that it has an averaging form, i.e. that $\hat{K}_{1}^{\epsilon}(x-y) \rightarrow \kappa_{1} \delta(x-y)$ as $\epsilon \rightarrow 0$. Common choices for $\hat{K}_{1}^{\epsilon} / \gamma$ are given in Remark 2.4, and include the popular Doi model in which the two reactants may react with a fixed probability per time when within $\epsilon$ [TS67, D76a, D76b]. We denote by $m_{1}(z \mid x, y)$ the probability density that when an A molecule at $x$ and a $\mathrm{B}$ molecule at $y$ react they produce at $\mathrm{C}$ molecule at $z \cdot m_{1}$ is commonly chosen to place $z$ on the line connecting $x$ and $y$, see Assumption 2.8. For the reverse reaction we assume $K_{2}(z)=\kappa_{2}$ denotes the constant probability per time an individual $\mathrm{C}$ molecule splits into $\mathrm{A}$ and $\mathrm{B}$ molecules, with $m_{2}^{\epsilon}(x, y \mid z)$ denoting the probability density that a $\mathrm{C}$ at $z$ which dissociates produces an $\mathrm{A}$ molecule at $x$ and a $\mathrm{B}$ molecule at $y$. The dependence of $m_{2}^{\epsilon}$ on $\epsilon$ follows under the assumption of detailed balance for the reversible reaction, see Assumption 2.10 and Assumption 2.11.

With these choices, the large population limit for the volume reactivity PBSRD model of the particles diffusing and reacting, a special case of the limit we proved for general systems in [IMS20], shows that the molar concentration fields for each species converge in a weak sense to the solution of a nonlocal system of PIDEs. More concretely, denote by $A(t)$ the stochastic process for the number of species A molecules at time $t$, and label the position of $i$ th molecule of species A at time $t$ by the stochastic process $\boldsymbol{Q}_{i}^{A(t)} \subset \mathbb{R}^{d}$. The random measure

$$
A_{\epsilon}^{\gamma}(x, t)=\frac{1}{\gamma} \sum_{i=1}^{A(t)} \delta\left(x-\boldsymbol{Q}_{i}^{A(t)}\right)
$$

corresponds to the stochastic process for the molar concentration of species A at $x$ at time $t$. We can similarly define $B_{\epsilon}^{\gamma}(x, t)$ and $C_{\epsilon}^{\gamma}(x, t)$. The large population (thermodynamic) limit where $\gamma \rightarrow \infty$ and $\left(A_{\epsilon}^{\gamma}(x, 0), B_{\epsilon}^{\gamma}(x, 0), C_{\epsilon}^{\gamma}(x, 0)\right)$ converge to well-defined limiting molar concentration fields gives (in a weak sense) that

$$
\left(A_{\epsilon}^{\gamma}(x, t), B_{\epsilon}^{\gamma}(x, t), C_{\epsilon}^{\gamma}(x, t)\right) \rightarrow\left(A_{\epsilon}(x, t), B_{\epsilon}(x, t), C_{\epsilon}(x, t)\right),
$$

which satisfy the MFM

$$
\begin{aligned}
& \partial_{t} A_{\epsilon}(x, t)=D_{1} \Delta_{x} A_{\epsilon}(x, t)-\left(\int_{\mathbb{R}^{d}} K_{1}^{\epsilon}(x-y) B_{\epsilon}(y, t) d y\right) A_{\epsilon}(x, t)+\int_{\mathbb{R}^{d}} K_{2}(z)\left(\int_{\mathbb{R}^{d}} m_{2}^{\epsilon}(x, y \mid z) d y\right) C_{\epsilon}(z, t) d z \\
& \partial_{t} B_{\epsilon}(y, t)=D_{2} \Delta_{y} B_{\epsilon}(y, t)-\left(\int_{\mathbb{R}^{d}} K_{1}^{\epsilon}(x-y) A_{\epsilon}(x, t) d x\right) B_{\epsilon}(y, t)+\int_{\mathbb{R}^{d}} K_{2}(z)\left(\int_{\mathbb{R}^{d}} m_{2}^{\epsilon}(x, y \mid z) d x\right) C_{\epsilon}(z, t) d z \\
& \partial_{t} C_{\epsilon}(z, t)=D_{3} \Delta_{z} C_{\epsilon}(z, t)-K_{2}(z) C_{\epsilon}(z, t)+\int_{\mathbb{R}^{2 d}} K_{1}(x, y) m_{1}(z \mid x, y) A_{\epsilon}(x, t) B_{\epsilon}(y, t) d x d y .
\end{aligned}
$$

The commonly used SM is a more formally derived system of reaction-diffusion PDEs. Denote by $A(x, t), B(y, t), C(z, t)$ the molar concentration fields for species $\mathrm{A}, \mathrm{B}, \mathrm{C}$ in the $\mathbf{S M}$, which satisfy

$$
\begin{aligned}
& \partial_{t} A(x, t)=D_{1} \Delta_{x} A(x, t)-\kappa_{1} A(x, t) B(x, t)+\kappa_{2} C(x, t), \\
& \partial_{t} B(y, t)=D_{2} \Delta_{y} B(y, t)-\kappa_{1} A(y, t) B(y, t)+\kappa_{2} C(z, t),
\end{aligned}
$$




$$
\partial_{t} C(z, t)=D_{3} \Delta_{z} C(z, t)-\kappa_{2} C(z, t)+\kappa_{1} A(z, t) B(z, t) .
$$

The main theoretical result of this paper, Theorem 3.1, proves that when the kernels and placement measures have averaging forms,

$$
\left(A_{\epsilon}(x, t), B_{\epsilon}(x, t), C_{\epsilon}(x, t)\right)=(A(x, t), B(x, y), C(x, t))+O\left(\epsilon^{2}\right), \quad \epsilon \rightarrow 0 .
$$

This demonstrates that we may interpret the $\mathbf{S M}$ as an approximation to the rigorous MFM when reactive interactions are short-range.

Nonlocal reaction-diffusion PIDE models related to our MFM have been introduced and studied for a variety of physical applications, including for models of population dynamics, evolutionary dynamics, and neuronal dynamics [NTY17, CFF19, SVB13, JG89, SSS20, PGB19, GB96]. These works focus on the effects of different nonlocal reaction kernels, and how the stability of steady states is influenced by the nonlocal interactions. To our knowledge, there is no rigorous analysis on the closeness between such nonlocal PIDE models and corresponding local reaction-diffusion models analogous to our SM. Our work is also distinguished in the generality of the considered chemical reaction systems, the range of spatial reaction rate kernels, and the variety of reaction product placement measures that are allowed.

As we completed this work, we became aware of the recent preprint [K20]. The authors provide an intuitive, formal argument for going from the Doi PBSRD model to the SM in the large-population limit for systems with bimolecular reactions. This involves discretizing the forward equation for the PBSRD model to a spatially-discrete convergent reaction-diffusion master equation (CRDME) model [I13, IZ18], assuming that the CRDME's second moments can be approximated as products of first moments in the large-population limit, and assuming that interaction length scales are sufficiently small that convolution sums representing bimolecular interactions between molecules at nearby lattice locations can be approximated by point interactions. Under these assumptions, a PDE corresponding to the SM for a bimolecular reaction is obtained when the lattice spacing within an approximating mean-field model for the CRDME is taken to zero. The present work is distinguished from such studies through our rigorous and direct proof that the SM is the short (bimolecular) interaction-range limit of the MFM (which was previously proven to be the rigorous large-population limit of the PBSRD model [LLN19, IMS20]). It is also distinguished in providing an error bound that demonstrates the rate of convergence of the MFM to the SM as the bimolecular interaction-distance is decreased.

Finally, we reiterate that in [IMS20] we proved that the MFM is the correct large population (thermodynamic) limit as $\gamma \rightarrow \infty$ of a wide-class of particle-based stochastic reaction diffusion models. In contrast, this paper explores the rigorous relationship between the $\mathbf{S M}$ and the $\mathbf{M F M}$ as $\epsilon$ is varied as explained above. The rest of the paper is organized as follows. In Section 2 we introduce the general setup, notation and our assumptions. In Section 3 we present our main result, Theorem 3.1, on the approximation of the MFM by the SM as the bimolecular reactive interaction distance $\epsilon \rightarrow 0$. In addition, we also present a number of numerical studies that demonstrate the (rate of) convergence of the MFM to the SM as $\epsilon \rightarrow 0$ (when both exist), and illustrate how well the MFM and SM models agree with the underlying PBSRD model for varying values of $\epsilon$. Our numerical results first demonstrate in several simple systems the theoretical findings that the $\mathbf{S M}$ is the short (bimolecular) interaction-range limit of the MFM, see Section 3.2. We then explore several biologically-motivated examples, see Section 3.3, where the MFM correctly captures essential behavior of the underlying particle-based stochastic system, but the SM sometimes fails to do so (or it is not immediately clear how to even define the SM). These illustrate the utility in using a rigorously derived-MFM for both model formulation when the SM is not clear, and as a means of assessing the accuracy of the SM. Section 4 proves local well-posedness of the MFM and the SM models. Section 5 discusses global well-posedness of the MFM for specific choices of the reaction kernels. Appendices A and B give proofs of technical results used throughout the paper.

\section{General Setup and Main Assumptions}

For simplicity, in our rigorous studies we assume that molecules diffuse freely in $\mathbb{R}^{d}$, with $D_{j}$ denoting the diffusion coefficient for species $S_{j}, j=1, \cdots, J$. Let $L$ be the number of possible reactions, each labelled by $\mathcal{R}_{1}, \cdots, \mathcal{R}_{L}$. The change in the number of particles induced by the $\mathcal{R}_{\ell}$ th reaction, $\ell \in\{1, \ldots, L\}$, is given by 
the chemical equation

$$
\sum_{j=1}^{J} \alpha_{\ell j} S_{j} \rightarrow \sum_{j=1}^{J} \beta_{\ell j} S_{j},
$$

where we assume the stoichiometric coefficients $\left\{\alpha_{\ell j}\right\}_{j=1}^{J}$ and $\left\{\beta_{\ell j}\right\}_{j=1}^{J}$ are non-negative integers. Let $\boldsymbol{\alpha}^{(\ell)}=$ $\left(\alpha_{\ell 1}, \alpha_{\ell 2}, \cdots, \alpha_{\ell J}\right)$ and $\boldsymbol{\beta}^{(\ell)}=\left(\beta_{\ell 1}, \beta_{\ell 2}, \cdots, \beta_{\ell J}\right)$ be multi-index vectors collecting the coefficients of the $\ell$ th reaction. We denote the reactant and product orders of the reaction by $\left|\boldsymbol{\alpha}^{(\ell)}\right| \doteq \sum_{i=1}^{J} \alpha_{\ell i} \leq 2$ and $\left|\boldsymbol{\beta}^{(\ell)}\right| \doteq \sum_{j=1}^{J} \beta_{\ell j} \leq 2$, assuming that at most two reactants and two products participate in any reaction. We therefore implicitly assume all reactions are at most second order.

We introduce two notations to encode reactant and product particle positions, that are subsequently needed to specify the MFM. Note, these definitions are the same under which we derived the MFM in [IMS20].

Definition 2.1. For reaction $\mathcal{R}_{\ell}$ we define the reactant position space

$$
\mathbb{X}^{(\ell)}=\left\{\boldsymbol{x}=\left(x_{1}^{(1)}, \cdots, x_{\alpha_{\ell 1}}^{(1)}, \cdots, x_{1}^{(J)}, \cdots, x_{\alpha_{\ell J}}^{(J)}\right) \mid x_{r}^{(j)} \in \mathbb{R}^{d}, \text { for all } 1 \leq j \leq J, 1 \leq r \leq \alpha_{\ell j}\right\}=\left(\mathbb{R}^{d}\right)^{\left|\boldsymbol{\alpha}^{(\ell)}\right|} .
$$

Here when $\alpha_{\ell j}=0$ species $j$ is not a reactant for the $\ell$ th reaction, and hence there will be no indices for particles of species $j$ within the reactant position space. In the underlying PBSRD model, $\boldsymbol{x} \in \mathbb{X}^{(\ell)}$ represents one possible configuration for the (sampled) positions of individual reactant particles that might undergo an $\mathcal{R}_{\ell}$ reaction. $x_{r}^{(j)}$ then labels the sampled position for the rth reactant particle of species $j$ involved in this specific instance of the reaction. We let $d \boldsymbol{x}=\left(\bigwedge_{j=1}^{J}\left(\bigwedge_{r=1}^{\alpha_{\ell_{j}}} d x_{r}^{(j)}\right)\right)$ be the corresponding volume form on $\mathbb{X}^{(\ell)}$.

Definition 2.2. For reaction $\mathcal{R}_{\ell}$ with $1 \leq \ell \leq L$ i.e. having at least one product particle we define the product position space

$$
\mathbb{Y}^{(\ell)}=\left\{\boldsymbol{y}=\left(y_{1}^{(1)}, \cdots, y_{\beta_{\ell 1}}^{(1)}, \cdots, y_{1}^{(J)}, \cdots, y_{\beta_{\ell J}}^{(J)}\right) \mid y_{r}^{(j)} \in \mathbb{R}^{d}, \text { for all } 1 \leq j \leq J, 1 \leq r \leq \beta_{\ell j}\right\}=\left(\mathbb{R}^{d}\right)^{\left|\boldsymbol{\beta}^{(\ell)}\right|} .
$$

Here when $\beta_{\ell j}=0$ species $j$ is not a product for the $\ell$ th reaction, and hence there will be no indices for particles of species $j$ within the product position space. In the underlying PBSRD model, $\boldsymbol{y} \in \mathbb{Y}^{(\ell)}$ represents one possible configuration for the (sampled) positions of individual product particles that might be produced by an $\mathcal{R}_{\ell}$ reaction. $y_{r}^{(j)}$ then labels the sampled position for the rth product particle of species $j$ involved in this specific instantance of the reaction. Let $d \boldsymbol{y}=\left(\bigwedge_{j=1}^{J}\left(\bigwedge_{r=1}^{\beta_{\ell j}} d y_{r}^{(j)}\right)\right)$ be the corresponding volume form on $\mathbb{Y}^{(\ell)}$.

Remark 2.1. In the case of no product for reaction $\mathcal{R}_{\ell}$ with $1 \leq \ell \leq L$, for example, $A \rightarrow \emptyset, A+B \rightarrow \emptyset$, etc, we naturally set the product position space $\mathbb{Y}^{(\ell)}$ defined in Definition 2.2 to be the empty set.

We denote by $K_{\ell}(\boldsymbol{x})$ the reaction rate kernel (i.e. probability per time) that reactant particles with positions $\boldsymbol{x} \in \mathbb{X}^{(\ell)}$ undergo reaction $\mathcal{R}_{\ell} . m_{\ell}(\boldsymbol{y} \mid \boldsymbol{x})$ will represent the reaction's placement measure, giving the probability density that when the reaction occurs reactants at positions $\boldsymbol{x} \in \mathbb{X}^{(\ell)}$ generate products at positions $\boldsymbol{y} \in \mathbb{Y}^{(\ell)}$.

We formulate the dynamics of the MFM in terms of the time evolution of the spatial molar concentration field for species $j$ at time $t$, denoted by $\rho_{j}(x, t), j=1,2, \cdots, J$. As summarized in the introduction, in [IMS20] we proved the coarse-grained large-population limit of the PBSRD model of particles diffusing and reacting is given by a coarse-grained system of PIDEs corresponding to our MFM. In contrast to the SM, the latter accounts for spatially distributed chemical interactions between particles in a manner that is consistent with the PBSRD model. For a general system of reacting and diffusing particles with the notation defined above, the derived coarse-grained MFM is given by the coupled system of (non-local) reaction-diffusion PIDEs

$$
\begin{aligned}
\partial_{t} \rho_{j}(x, t)= & D_{j} \Delta_{x} \rho_{j}(x, t)-\sum_{\ell=1}^{L}\left(\frac{1}{\boldsymbol{\alpha}^{(\ell) !}} \sum_{r=1}^{\alpha_{\ell j}} \int_{\tilde{\boldsymbol{x}} \in \mathbb{X}^{(\ell)}} \delta_{x}\left(\tilde{x}_{r}^{(j)}\right) K_{\ell}(\tilde{\boldsymbol{x}})\left(\Pi_{k=1}^{J} \Pi_{s=1}^{\alpha_{\ell k}} \rho_{k}\left(\tilde{x}_{s}^{(k)}, t\right)\right) d \tilde{\boldsymbol{x}}\right) . \\
& +\sum_{\ell=1}^{L}\left(\frac{1}{\boldsymbol{\alpha}^{(\ell) !}} \sum_{r=1}^{\beta_{\ell j}} \int_{\tilde{\boldsymbol{x}} \in \mathbb{X}^{(\ell)}} K_{\ell}(\tilde{\boldsymbol{x}})\left(\int_{\boldsymbol{y} \in \mathbb{Y}^{(\ell)}} \delta_{x}\left(y_{r}^{(j)}\right) m_{\ell}(\boldsymbol{y} \mid \tilde{\boldsymbol{x}}) d \boldsymbol{y}\right)\left(\Pi_{k=1}^{J} \Pi_{s=1}^{\alpha_{\ell k}} \rho_{k}\left(\tilde{x}_{s}^{(k)}, t\right)\right) d \tilde{\boldsymbol{x}}\right),
\end{aligned}
$$


where $j=1, \ldots, J$.

Remark 2.2. In Eq. (2.1) the expressions $\int \delta_{x}\left(\tilde{x}_{r}^{(j)}\right) \cdots d \tilde{x}_{r}^{(j)}$ and $\int \delta_{x}\left(y_{r}^{(j)}\right) \cdots d y_{r}^{(j)}$ are used as convenient and systematic notations to represent replacing $\tilde{x}_{r}^{(j)}$ and $y_{r}^{(j)}$ with $x$ through the formal action of the $\delta$ function. That is

$$
\int_{\mathbb{R}^{d}} \delta_{x}\left(\tilde{x}_{r}^{(j)}\right) f\left(\tilde{x}_{r}^{(j)}\right) d \tilde{x}_{r}^{(j)} \doteq f(x) .
$$

In contrast to the MFM, the standard reaction-diffusion PDE model (SM) used for modeling the time evolution of chemical concentration fields extends spatially-homogeneous law of mass action-based ODE models by adding Laplacians to model molecular diffusion [ECM07, MNKS09, NTS08]. The SM involves only local chemical interactions, a major simplification from more-detailed PBSRD models, with reactions occurring based on a spatially uniform reaction rate constant inherited from the underlying mass-action ODE model. For reaction $\mathcal{R}_{\ell}$ we denote this constant by $\kappa_{\ell}$. The $\mathbf{S M}$ is then the coupled system of PDEs

$$
\partial_{t} \rho_{j}(x, t)=D_{j} \Delta_{x} \rho_{j}(x, t)+\sum_{\ell=1}^{L} \kappa_{\ell}\left(\beta_{\ell j}-\alpha_{\ell j}\right)\left(\Pi_{k=1}^{J} \rho_{k}(x, t)^{\alpha_{\ell k}}\right), \quad j=1, \ldots, J .
$$

2.1. Assumptions for MFM. To discuss the connection between the MFM and SM models, we introduce a reactive interaction scale parameter $\epsilon$ in the following assumptions on the reaction kernels and placement measures for the MFM. In the remainder, we will exploit that in many applications $\epsilon$ is a small compared to macroscopic length-scales over which we are interested in the dynamics of the concentration fields.

Assumption 2.3. We assume that for bimolecular reactions the rate kernels $K_{\ell}(x, y), x, y \in \mathbb{R}^{d}$ depend only on the separation distance, $|x-y|$, of the two particles. In particular, we shall write $K_{\ell}(x, y)=K_{\ell}^{\epsilon}(x, y)=$ $\hat{K}_{\ell}^{\epsilon}(x-y)=\hat{K}_{\ell}^{\epsilon}(y-x)$, where $\hat{K}$ has domain $\mathbb{R}^{d}$, to indicate this more explicitly, and to indicate the length scale over which the reaction can occur. We also assume that $\left\|\hat{K}_{\ell}^{\epsilon}(w)\right\|_{L^{1}}=k_{\ell}$ and $\int_{\mathbb{R}^{d}} \hat{K}_{\ell}^{\epsilon}(w)|w|^{2} d w=O\left(\epsilon^{2}\right)$ for all $\epsilon$. This last assumption is key for determining the rate of convergence of the $\boldsymbol{M F M}$ to the $\boldsymbol{S M}$ we establish in Theorem 3.1 as $\epsilon \rightarrow 0$. Finally, as $\epsilon \rightarrow 0$ we assume that $\hat{K}_{\ell}^{\epsilon}(w) \rightarrow k_{\ell} \delta(w)$ in distribution.

Remark 2.4. We previously showed how such $\epsilon$-scalings arise naturally when calibrating parameters in the PBSRD model to recover known/measured well-mixed parameters in the fast diffusion limit [IMS20]. For example, with such calibrations Assumption 2.3 is satisfied for the commonly used Doi kernel

$$
\hat{K}_{\ell}^{\epsilon}(w)=\frac{k_{\ell}}{\left|B_{\epsilon}(0)\right|} \mathbb{1}_{\{|w| \leq \epsilon\}},
$$

where $\left|B_{\epsilon}(0)\right|$ is the volume of d-dimensional ball with radius $\epsilon$. The scaling can also be derived from more microscopic polymer models of tethered interactions between membrane bound proteins, which give the coarsegrained Gaussian kernel

$$
\hat{K}_{\ell}^{\epsilon}(w)=\frac{k_{\ell}}{\left(\sqrt{2 \pi \epsilon^{2}}\right)^{d}} e^{-\frac{|w|^{2}}{2 \epsilon^{2}}}
$$

see [ZI20] for details and references.

Both these kernels are examples with a mollification-type scaling that $\hat{K}_{\ell}^{\epsilon}(w)=\frac{1}{\epsilon^{d}} \hat{K}_{\ell}^{1}\left(\frac{w}{\epsilon}\right)$, with $\left\|\hat{K}_{\ell}^{1}(w)\right\|_{L^{1}}=$ $k_{\ell}$ and a finite second moment (i.e. $\left.\left\|\hat{K}_{\ell}^{1}(w)|w|^{2}\right\|_{L^{1}}<\infty\right)$. We then have that $\int_{\mathbb{R}^{d}} \hat{K}_{\ell}^{\epsilon}(w)|w|^{2} d w=o\left(\epsilon^{2}\right)$ as $\epsilon \rightarrow 0$, and $\hat{K}_{\ell}^{\epsilon}(w) \rightarrow k_{\ell} \delta(w)$ in distribution as $\epsilon \rightarrow 0$, motivating our choices in Assumption 2.3.

Assumption 2.5. We assume that the unimolecular reaction rate function $K_{\ell}(x), x \in \mathbb{R}^{d}$, is a constant i.e. $K_{\ell}(x)=k_{\ell}$. We note that there is no $\epsilon$ dependence in this case.

Assumption 2.6. We assume that for any $1 \leq \ell \leq L, \boldsymbol{y} \in \mathbb{Y}^{(\ell)}$ and $\boldsymbol{x} \in \mathbb{X}^{(\ell)}$, the placement measure $m_{\ell}(\boldsymbol{y} \mid \boldsymbol{x})$ is a probability measure, i.e. $\int_{\mathbb{Y}(\ell)} m_{\ell}(\boldsymbol{y} \mid \boldsymbol{x}) d \boldsymbol{y}=1$. Notice that in the case of a reaction with no products, $\mathbb{Y}^{(\ell)}=\emptyset$, without loss of generality, let us assume that $\int_{\mathbb{Y}^{(\ell)}} m_{\ell}(\boldsymbol{y} \mid \boldsymbol{x}) d \boldsymbol{y}=1$ still holds.

If $\mathcal{R}_{\ell}$ is a zeroth order reaction (birth reaction) of the form $\emptyset \rightarrow S_{i}$, the reaction rate function is typically assumed to be a constant, $K_{\ell}=k_{\ell}$. In [IMS20] (Remark 5.7), we explained how the mean-field largepopulation limit holds if we assume that the placement measure $m(y), y \in \mathbb{R}^{d}$ for such a birth reaction has 
compact support. In biological applications such reactions typically occur within a compact region of $\mathbb{R}^{3}$, for example the interior of a cell, and as such $K_{\ell}$ and the placement measure should be zero outside of the region of interest. To avoid having a spatially varying birth rate, in the remainder we exclude zero'th order birth processes. We note, however, that this choice is made to simplify notation and subsequent calculations; nothing in our analysis fundamentally precludes the incorporation of zeroth order reactions.

Assumption 2.7. If $\mathcal{R}_{\ell}$ is a first order reaction of the form $S_{i} \rightarrow S_{j}$, we assume that the placement measure $m_{\ell}(y \mid x)$ takes the form

$$
m_{\ell}(y \mid x)=\delta_{x}(y) .
$$

This describes that the newly created $S_{j}$ particle is placed at the position of the reactant $S_{i}$ particle.

Assumption 2.8. If $\mathcal{R}_{\ell}$ is a second order reaction of the form $S_{i}+S_{k} \rightarrow S_{j}$, we assume that the placement measure $m_{\ell}(z \mid x, y)$ takes the form

$$
m_{\ell}(z \mid x, y)=\sum_{i=1}^{I} p_{i} \times \delta\left(z-\left(\alpha_{i} x+\left(1-\alpha_{i}\right) y\right)\right),
$$

where $I$ is a fixed finite integer and $\sum_{i} p_{i}=1$. This describes that the creation of particle $S_{j}$ is always on the segment connecting the reactant $S_{i}$ and reactant $S_{k}$ particles, but allows some random choice of position. $A$ special case would be $I=2, p_{i}=\frac{1}{2}, \alpha_{1}=0$ and $\alpha_{2}=1$, which corresponds to placing the particle randomly at the position of one of the two reactants. One common choice is taking $I=1, p_{1}=1$ and choosing $\alpha_{1}$ to be the diffusion weighted center of mass [IZ18].

Assumption 2.9. If $\mathcal{R}_{\ell}$ is a second order reaction of the form $S_{i}+S_{k} \rightarrow S_{j}+S_{r}$, we assume that the placement measure $m_{\ell}(z, w \mid x, y)$ takes the form

$$
m_{\ell}(z, w \mid x, y)=p \times \delta_{(x, y)}((z, w))+(1-p) \times \delta_{(x, y)}((w, z)) .
$$

This describes that newly created product $S_{j}$ and $S_{r}$ particles are always at the positions of the reactant $S_{i}$ and $S_{k}$ particles. $p$ is typically either 0 or 1 , depending on the underlying physics of the reaction.

Assumption 2.10. If $\mathcal{R}_{\ell}$ is a first order reaction of the form $S_{i} \rightarrow S_{j}+S_{k}$, we assume the placement measure depends on the separation scale parameter $\epsilon$ and is in the form

$$
m_{\ell}^{\epsilon}(x, y \mid z)=\rho^{\epsilon}(|x-y|) \sum_{i=1}^{I} p_{i} \times \delta\left(z-\left(\alpha_{i} x+\left(1-\alpha_{i}\right) y\right)\right),
$$

with $\sum_{i} p_{i}=1$. Here we assume the relative separation of the product $S_{j}$ and $S_{k}$ particles, $|x-y|$, is sampled from the probability density $\rho^{\epsilon}(|x-y|)$. Their (weighted) center of mass is sampled from the density encoded by the sum of $\delta$ functions. Such forms are common for detailed balance preserving reversible bimolecular reactions [IZ18], from which $\rho^{\epsilon}$ obtains the explicit $\epsilon$ dependence.

We further assume some regularity of the separation placement density, $\rho^{\epsilon}(|w|), w \in \mathbb{R}^{d}$, introduced in Assumption 2.10:

Assumption 2.11. For Assumption 2.6 to be true, we require $\left\|\rho^{\epsilon}\right\|_{L^{1}\left(\mathbb{R}^{d}\right)}=1$ for all $\epsilon$. When $\rho^{\epsilon}(|w|)$ comes from a reversible bimolecular reaction that satisfies detailed-balance, it will have a similar functional form to the bimolecular reaction kernel $\hat{K}_{\ell}^{\epsilon}(w)$ in Assumption 2.3. For this reason, as $\epsilon \rightarrow 0$ we assume that $\rho^{\epsilon}(|w|) \rightarrow \delta(w)$ in distribution and $\int_{\mathbb{R}^{d}} \rho^{\epsilon}(|w|)|w|^{2} d w=O\left(\epsilon^{2}\right)$.

Note, with the preceding assumptions the placement measure only depends on $\epsilon$ for dissociation reactions of the form $S_{i} \rightarrow S_{j}+S_{k}$.

In what follows we rewrite the MFM to make explicit the $\epsilon$-dependence giving

$$
\begin{aligned}
\partial_{t} \rho_{j}^{\epsilon}(x, t)= & D_{j} \Delta_{x} \rho_{j}^{\epsilon}(x, t)-\sum_{\ell=1}^{L}\left(\frac{1}{\boldsymbol{\alpha}^{(\ell) !}} \sum_{r=1}^{\alpha_{\ell j}} \int_{\tilde{\boldsymbol{x}} \in \mathbb{X}(\ell)} \delta_{x}\left(\tilde{x}_{r}^{(j)}\right) K_{\ell}^{\epsilon}(\tilde{\boldsymbol{x}})\left(\Pi_{k=1}^{J} \Pi_{s=1}^{\alpha_{\ell k}} \rho_{k}^{\epsilon}\left(\tilde{x}_{s}^{(k)}, t\right)\right) d \tilde{\boldsymbol{x}}\right) \\
& +\sum_{\ell=1}^{L}\left(\frac{1}{\boldsymbol{\alpha}^{(\ell) !}} \sum_{r=1}^{\beta_{\ell j}} \int_{\tilde{\boldsymbol{x} \in \mathbb{X}(\ell)}} K_{\ell}^{\epsilon}(\tilde{\boldsymbol{x}})\left(\int_{\boldsymbol{y} \in \mathbb{Y}(\ell)} \delta_{x}\left(y_{r}^{(j)}\right) m_{\ell}^{\epsilon}(\boldsymbol{y} \mid \tilde{\boldsymbol{x}}) d \boldsymbol{y}\right)\left(\Pi_{k=1}^{J} \Pi_{s=1}^{\alpha_{\ell k}} \rho_{k}^{\epsilon}\left(\tilde{x}_{s}^{(k)}, t\right)\right) d \tilde{\boldsymbol{x}}\right)
\end{aligned}
$$

for $j=1, \ldots, J$. 


\section{MAin Results}

With the preceding assumptions, we now prove the rigorous relationship between the MFM and SM models as the reactive interaction scale $\epsilon \rightarrow 0$. Our main theoretical result is given by Theorem 3.1 on the approximation of the MFM, Eq. (2.3), by the SM, Eq. (2.2) as $\epsilon \rightarrow 0$. In addition, we present a series of numerical studies in one and two dimensions to demonstrate the relationship between the two models and the underlying PBSRD model.

Before proceeding with Theorem 3.1, let us briefly discuss the issue of well-posedness of models Eq. (2.1) (equivalently Eq. (2.3)) and Eq. (2.2). The mild solution to both models is of the form

$$
\boldsymbol{\rho}(t)=S(t) \boldsymbol{\rho}(0)+\int_{0}^{t} S(t-s) N[\boldsymbol{\rho}](s) d s,
$$

where $\boldsymbol{\rho}(t)=\left(\rho_{1}(\cdot, t), \rho_{2}(\cdot, t), \cdots, \rho_{J}(\cdot, t)\right)^{T}, S(t)$ is the semigroup generated by the linear diffusion operator $\operatorname{Diag}\left(D_{1} \Delta_{x}, D_{2} \Delta_{x}, \cdots, D_{J} \Delta_{x}\right)$, and $N[\boldsymbol{\rho}]=\left((N[\boldsymbol{\rho}])_{1},(N[\boldsymbol{\rho}])_{2}, \cdots,(N[\boldsymbol{\rho}])_{J}\right)^{T}$ represents the nonlinear reaction term.

Depending on the properties of the nonlinear reaction term $N[\boldsymbol{\rho}]$ one obtains local in time well-posedness, i.e. in some interval $\left[0, T_{0}\right]$, or one obtains global in time well-posedness. In Section 4 we discuss local in time well posedness and regularity for both equations Eq. (2.1) and Eq. (2.2). In Section 5, we discuss under which additional assumptions on $N[\boldsymbol{\rho}]$ one has global well-posedness. As illustrative examples, in Section 5 we prove that reaction systems with reactions of the type $A+B \leftrightarrows C+D$ and $A+B \leftrightarrows C$ both satisfy the requirements for global well-posedness (the latter under specific choices for the placement measure).

3.1. Approximation theorem. Let $C_{b, \text { unif }}\left(\mathbb{R}^{d}\right)$ denote the space of bounded and uniformly continuous functions on $\mathbb{R}^{d}$, and denote by $C_{b}^{\ell}\left(\mathbb{R}^{d}\right)$ the space of functions with continuous and uniformly bounded derivatives on $\mathbb{R}^{d}$ through order $\ell$. Our main result is the following theorem on the convergence of the MFM to the SM as $\epsilon \rightarrow 0$.

Theorem 3.1. Let $T_{0}$ be a time such that the solutions to the $\boldsymbol{S M}$ and $\boldsymbol{M F M}$ are uniformly bounded for $(x, t) \in \mathbb{R}^{d} \times\left[0, T_{0}\right]$ and $\epsilon$. Let $\rho_{j}(x, 0)=\rho_{j}^{\epsilon}(x, 0) \in C_{b}^{1}\left(\mathbb{R}^{d}\right) \cap C_{b, \text { unif }}\left(\mathbb{R}^{d}\right)$ for all $\epsilon>0$ and $j=1, \cdots, J$, and assume that

$$
k_{\ell}=\left(\boldsymbol{\alpha}^{(\ell)} !\right) \kappa_{\ell}
$$

Under the assumptions in Section 2.1 we have that the solution to Eq. (2.3) converges uniformly in $(x, t)$ to the solution to Eq. (2.2) at second order as $\epsilon \rightarrow 0$, i.e.

$$
\sup _{t \in\left[0, T_{0}\right]} \max _{j=1, \cdots, J}\left\|\rho_{j}(x, t)-\rho_{j}^{\epsilon}(x, t)\right\|_{L^{\infty}}=O\left(\epsilon^{2}\right) .
$$

Remark 3.2. One can find such a $T_{0}$ in Theorem 3.1 by a contraction mapping approach as in Section 4, or choose any $T_{0}<\infty$ for particular reaction systems for which global well-posedness holds with appropriate uniform estimates. Systems of the form $A+B \leftrightarrows C+D$ or $A+B \leftrightarrows C$ are shown to have such global well-posedness estimates in Section 5. More generally, whether $T_{0}$ can be chosen arbitrarily large will depend on the global well-posedness of the $\boldsymbol{M F M}$ and the $\boldsymbol{S} \boldsymbol{M}$ for a particular reaction network. As we describe in Section 5, the construction of such a global well-posedness theory for general reaction systems is a still an open problem.

Proof. By taking the difference of the mild solution to Eq. (2.3) and Eq. (2.2) for any species $j, 1 \leq j \leq J$, we have that

$$
\begin{aligned}
& \rho_{j}(x, t)-\rho_{j}^{\epsilon}(x, t)=e^{t D_{j} \Delta_{x}}\left(\rho_{j}(x, 0)-\rho_{j}^{\epsilon}(x, 0)\right)-\sum_{\ell=1}^{L} \int_{0}^{t} e^{(t-\tau) D_{j} \Delta_{x}} \sum_{r=1}^{\alpha_{\ell j}}\left[\kappa_{\ell} \times \Pi_{k=1}^{J} \Pi_{s=1}^{\alpha_{\ell k}} \rho_{k}(x, \tau)\right. \\
& \left.-\left(\frac{1}{\boldsymbol{\alpha}^{(\ell) !}} \int_{\tilde{\boldsymbol{x}} \in \mathbb{X}^{(\ell)}} \delta_{x}\left(\tilde{x}_{r}^{(j)}\right) K_{\ell}^{\epsilon}(\tilde{\boldsymbol{x}})\left(\Pi_{k=1}^{J} \Pi_{s=1}^{\alpha_{\ell k}} \rho_{k}^{\epsilon}\left(\tilde{x}_{s}^{(k)}, \tau\right)\right) d \tilde{\boldsymbol{x}}\right)\right] \\
& +\sum_{\ell=1}^{L} \int_{0}^{t} e^{(t-\tau) D_{j} \Delta_{x}} \sum_{r=1}^{\beta_{\ell j}}\left[\kappa_{\ell} \times \Pi_{k=1}^{J} \Pi_{s=1}^{\alpha_{\ell k}} \rho_{k}(x, \tau)\right.
\end{aligned}
$$




$$
\left.-\left(\frac{1}{\boldsymbol{\alpha}^{(\ell) !}} \int_{\tilde{\boldsymbol{x}} \in \mathbb{X}^{(\ell)}} K_{\ell}^{\epsilon}(\tilde{\boldsymbol{x}})\left(\int_{\boldsymbol{y} \in \mathbb{Y}^{(\ell)}} \delta_{x}\left(y_{r}^{(j)}\right) m_{\ell}^{\epsilon}(\boldsymbol{y} \mid \tilde{\boldsymbol{x}}) d \boldsymbol{y}\right)\left(\Pi_{k=1}^{J} \Pi_{s=1}^{\alpha_{\ell k}} \rho_{k}^{\epsilon}\left(\tilde{x}_{s}^{(k)}, \tau\right)\right) d \tilde{\boldsymbol{x}}\right)\right] d \tau .
$$

Using that $\left\|e^{t D_{j} \Delta_{x}} f\right\|_{L^{\infty}} \leq\|f\|_{L^{\infty}}$ for all $t \geq 0$ and $f \in L^{\infty}\left(\mathbb{R}^{d}\right)$, we have that

$$
\left\|\rho_{j}(x, t)-\rho_{j}^{\epsilon}(x, t)\right\|_{L^{\infty}} \leq\left\|\rho_{j}(x, 0)-\rho_{j}^{\epsilon}(x, 0)\right\|_{L^{\infty}}+\sum_{\ell=1}^{L} \int_{0}^{t} \Lambda^{\ell, j}(\tau)+\Theta^{\ell, j}(\tau) d \tau,
$$

where

$$
\Lambda^{\ell, j}(\tau)=\sum_{r=1}^{\alpha_{\ell j}}\left\|\left(\frac{1}{\boldsymbol{\alpha}^{(\ell) !}} \int_{\tilde{\boldsymbol{x}} \in \mathbb{X}(\ell)} \delta_{x}\left(\tilde{x}_{r}^{(j)}\right) K_{\ell}^{\epsilon}(\tilde{\boldsymbol{x}})\left(\Pi_{k=1}^{J} \Pi_{s=1}^{\alpha_{\ell k}} \rho_{k}^{\epsilon}\left(\tilde{x}_{s}^{(k)}, \tau\right)\right) d \tilde{\boldsymbol{x}}\right)-\kappa_{\ell} \times \Pi_{k=1}^{J} \Pi_{s=1}^{\alpha_{\ell k}} \rho_{k}(x, \tau)\right\|_{L^{\infty}},
$$

and

$$
\begin{array}{r}
\Theta^{\ell, j}(\tau)=\sum_{r=1}^{\beta_{\ell j}} \|\left(\frac{1}{\boldsymbol{\alpha}^{(\ell) !}} \int_{\tilde{\boldsymbol{x}} \in \mathbb{X}(\ell)} K_{\ell}^{\epsilon}(\tilde{\boldsymbol{x}})\left(\int_{\boldsymbol{y} \in \mathbb{Y}^{(\ell)}} \delta_{x}\left(y_{r}^{(j)}\right) m_{\ell}^{\epsilon}(\boldsymbol{y} \mid \tilde{\boldsymbol{x}}) d \boldsymbol{y}\right)\left(\Pi_{k=1}^{J} \Pi_{s=1}^{\alpha_{\ell k}} \rho_{k}^{\epsilon}\left(\tilde{x}_{s}^{(k)}, \tau\right)\right) d \tilde{\boldsymbol{x}}\right) \\
-\kappa_{\ell} \times \Pi_{k=1}^{J} \Pi_{s=1}^{\alpha_{\ell k}} \rho_{k}(x, \tau) \|_{L^{\infty}} .
\end{array}
$$

From now on, and also in Lemma A.3 and Lemma A.4, we will use the following notations for generic constants. Using the assumed uniform boundedness of the MFM and SM solutions, we have

$$
C:=\left(\max _{\ell=1, \cdots, L} k_{\ell}\right) \max _{j=1, \cdots, J}\left\{\sup _{\epsilon>0} \sup _{\tau \in\left[0, T_{0}\right]}\left\|\rho_{j}^{\epsilon}(x, \tau)\right\|_{L^{\infty}} \vee \sup _{\tau \in\left[0, T_{0}\right]}\left\|\rho_{j}(x, \tau)\right\|_{L^{\infty}}\right\}<\infty .
$$

(Inequality 1) in Theorem 4.4 and Theorem 4.6 then give that

$$
\max _{j=1, \cdots, J}\left\{\sup _{\epsilon>0} \sup _{\tau \in\left[0, T_{0}\right]}\left\|\rho_{j}^{\epsilon}(x, \tau)\right\|_{C_{b}^{1}\left(\mathbb{R}^{d}\right)} \vee \sup _{\tau \in\left[0, T_{0}\right]}\left\|\rho_{j}(x, \tau)\right\|_{C_{b}^{1}\left(\mathbb{R}^{d}\right)}\right\} \leq C_{1},
$$

for some constant $C_{1}$ depending only on $C, \sup _{j=1, \cdots, J}\left\|\rho_{j}(x, 0)\right\|_{C_{b}^{1}\left(\mathbb{R}^{d}\right)}$ and $T_{0}$. Similarly, (Inequality 2) in Theorem 4.4 and Theorem 4.6 give

$$
\max _{j=1, \cdots, J}\left\{\sup _{\epsilon>0}\left\|\rho_{j}^{\epsilon}(x, \tau)\right\|_{C_{b}^{2}\left(\mathbb{R}^{d}\right)} \vee\left\|\rho_{j}(x, \tau)\right\|_{C_{b}^{2}\left(\mathbb{R}^{d}\right)}\right\} \leq C_{2}+\frac{C_{3}}{\sqrt{\tau}} .
$$

Combined with Eq. (3.6), we have that

$$
\max _{j, k=1, \cdots, J}\left\{\sup _{\epsilon>0}\left\|\rho_{j}^{\epsilon}(x, \tau) \rho_{k}^{\epsilon}(x, \tau)\right\|_{C_{b}^{2}\left(\mathbb{R}^{d}\right)} \vee\left\|\rho_{j}(x, \tau) \rho_{k}(x, \tau)\right\|_{C_{b}^{2}\left(\mathbb{R}^{d}\right)}\right\} \leq C_{2}+\frac{C_{3}}{\sqrt{\tau}},
$$

for any fixed $\tau \in\left(0, T_{0}\right]$ and some (other) constants $C_{2}$ and $C_{3}$ only depending on $C, \sup _{j=1, \cdots, J}\left\|\rho_{j}(x, 0)\right\|_{C_{b}^{1}\left(\mathbb{R}^{d}\right)}$ and $T_{0}$.

Using the estimates from Lemma A.3 and Lemma A.4, Eq. (3.3) becomes

$$
\begin{aligned}
\left\|\rho_{j}(x, t)-\rho_{j}^{\epsilon}(x, t)\right\|_{L^{\infty}} \leq & \left\|\rho_{j}(x, 0)-\rho_{j}^{\epsilon}(x, 0)\right\|_{L^{\infty}} \\
& \quad+\sum_{\ell=1}^{L} \int_{0}^{t} 6 C\left(\max _{j=1, \cdots, J}\left\|\rho_{j}(x, \tau)-\rho_{j}^{\epsilon}(x, \tau)\right\|_{L^{\infty}}+\left(C_{2}+\frac{C_{3}}{\sqrt{\tau}}\right) O\left(\epsilon^{2}\right)\right) d \tau \\
\leq & \left\|\rho_{j}(x, 0)-\rho_{j}^{\epsilon}(x, 0)\right\|_{L^{\infty}}+6 C L\left(C_{2} t+2 C_{3} \sqrt{t}\right) O\left(\epsilon^{2}\right) \\
& +6 C L \int_{0}^{t}\left(\max _{j=1, \cdots, J}\left\|\rho_{j}(x, \tau)-\rho_{j}^{\epsilon}(x, \tau)\right\|_{L^{\infty}}\right) d \tau .
\end{aligned}
$$

Applying Gronwall's Lemma we have

$$
\max _{j=1, \cdots, J}\left\|\rho_{j}(x, t)-\rho_{j}^{\epsilon}(x, t)\right\|_{L^{\infty}} \leq\left(6 C L\left(C_{2} t+2 C_{3} \sqrt{t}\right) O\left(\epsilon^{2}\right)+\max _{j=1, \cdots, J}\left\|\rho_{j}(x, 0)-\rho_{j}^{\epsilon}(x, 0)\right\|_{L^{\infty}}\right) e^{6 C L t}
$$

so that

$$
\sup _{t \in\left[0, T_{0}\right]} \max _{j=1, \cdots, J}\left\|\rho_{j}(x, t)-\rho_{j}^{\epsilon}(x, t)\right\|_{L^{\infty}} \leq 6 C L\left(C_{2} T_{0}+2 C_{3} \sqrt{T_{0}}\right) e^{6 C L T_{0}} O\left(\epsilon^{2}\right) .
$$


3.2. Numerical Comparison for Reversible $A+B \rightleftarrows C$ Reaction. In order to illustrate Theorem 3.1 and further investigate the connections between the SM and the MFM, we numerically solved the reversible $A+B \rightleftarrows C$ reaction using each of the PBSRD model, the SM, and the MFM. The PDEs and PIDEs for the SM and MFM were solved in MATLAB with periodic boundary conditions on both the interval $[0, L]$, and the square $[0, L] \times[0, L]$. The stochastic process associated with the PBSRD model was numerically solved by discretization to a jump process via the Convergent Reaction Diffusion Master Equation (CRDME) [I13, IZ18], which was then sampled using the Gibson-Bruck stochastic simulation algorithm [GB200]. Due to the computational expense of the PBSRD model in the large-population limit, we only solved it for the one-dimensional problem with periodic boundary conditions on $[0, L]$.

Let us denote by $A(x, t), B(y, t), C(z, t)$ the concentration fields for species $\mathrm{A}, \mathrm{B}, \mathrm{C}$ respectively in the SM, Eq. (1.2).

Similarly, we denote by $A_{\epsilon}(x, t), B_{\epsilon}(y, t), C_{\epsilon}(z, t)$ the concentration fields for species A, B, C respectively in the MFM Eq. (2.3), with separation scale parameter $\epsilon$. The latter then satisfy Eq. (1.1).

In the following we fixed $L=2 \pi$, the diffusivity $D_{1}=1, D_{2}=0.5, D_{3}=0.1$, and assumed a detailed balanced condition on the reversible reactions, i.e.

$$
K_{d} \hat{K}_{1}^{\epsilon}(x-y) m_{1}(z \mid x, y)=K_{2}(z) m_{2}^{\epsilon}(x, y \mid z),
$$

where $K_{d}=k_{2} / k_{1}$ is the equilibrium dissociation constant of the reaction, see [IZ18]. We set $\kappa_{1}=k_{1}=$ $\left\|\hat{K}_{1}^{\epsilon}\right\|_{L^{1}\left(\mathbb{R}^{d}\right)}=1$ and $\kappa_{2}=k_{2}=K_{2}(z)=0.05$ for any $z \in \mathbb{R}^{d}$. We consider two reaction kernels: the Doi kernel

$$
\hat{K}_{1}^{\epsilon}(w)=\frac{k_{1}}{\left|B_{\epsilon}(0)\right|} \mathbb{1}_{\{|w| \leq \epsilon\}},
$$

where $\left|B_{\epsilon}(0)\right|$ is the volume of d-dimensional ball with radius $\epsilon$, and the Gaussian kernel

$$
\hat{K}_{1}^{\epsilon}(w)=\frac{k_{1}}{\left(\sqrt{2 \pi \epsilon^{2}}\right)^{d}} e^{-\frac{|w|^{2}}{2 \epsilon^{2}}},
$$

for any $w \in \mathbb{R}^{d}$.

The PDEs and PIDEs were solved using a Fourier collocation method [HGG07] for the spatial discretization, with collocation points $x_{i}=\frac{i L}{N}, i=0, \cdots, N-1$ in $1 \mathrm{~d}$ and $\left(x_{i}, y_{j}\right)=\left(\frac{i L}{N}, \frac{j L}{N}\right), i, j=0, \cdots, N-1$ in $2 \mathrm{~d}$. We chose $N=2^{9}$ for $1 \mathrm{~d}$ and $N=2^{8}$ for 2 d. We approximated integral terms in the MFM using the midpoint quadrature rule centered at collocation points. For the reaction-diffusion equations the diffusion terms were stiff whereas the reaction terms were non-stiff. We therefore used the Crank-Nicolson Adams-Bashforth (CNAB) implicit-explicit method (IMEX) to discretize in time the spatially discretized system, with a time step of $\Delta t=1 e-3$ (for stability and accuracy reasons). As CNAB is a two-step multistep method, to obtain the numerical solution at time $\Delta t$ we applied the one-step IMEX Forward-Backward Euler method with a time step of $(\Delta t)^{2}$ until time $\Delta t$.

3.2.1. One Dimensional Results. In the one dimensional periodic domain $[0, L]$, we set the initial conditions for both models to be $A(x, 0)=e^{-10(x-1)^{2}}, B(y, 0)=e^{-10(y-2)^{2}}$ and $C(z, 0)=0$.

We first examine the relationship between the PBSRD model, the MFM and the SM as shown in Figs. 1 and 2. It is clear that the molar concentration fields and molar masses in the MFM are a good approximation of the PBSRD model for $\gamma$, the large-population limit scaling parameter in the PBSRD model [IMS20], sufficiently large. For $\epsilon$ sufficiently small both the SM and MFM are good approximations, while for $\epsilon$ sufficiently large, only the MFM is a good approximation to the PBSRD model.

We next compared the SM to the MFM with various combinations of reaction kernels and displacement measures. In particular, we investigated the MFM with (1) Gaussian kernel and placement measure $m_{1}(z \mid x, y)=\delta(z-(.5 x+.5 y)),(2)$ Gaussian kernel and placement measure $m_{1}(z \mid x, y)=.5 \delta(z-x)+.5 \delta(z-y)$, (3) Doi kernel and placement measure $m_{1}(z \mid x, y)=.5 \delta(z-x)+.5 \delta(z-y)$. For all these choices of the reaction kernel and placement measure, the MFM PIDE solution converges to the SM PDE solution at second order in $\epsilon$ as shown in Fig. 3. This illustrates our rigorous results on the convergence proven in Theorem 3.1.

3.2.2. Two Dimensional Results. We further tested the convergence over a two dimensional periodic domain, comparing the SM PDE solution to the MFM PIDE solution for a representative Gaussian reaction kernel 


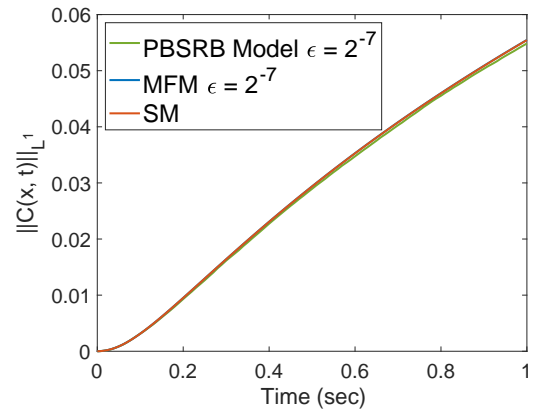

(a)

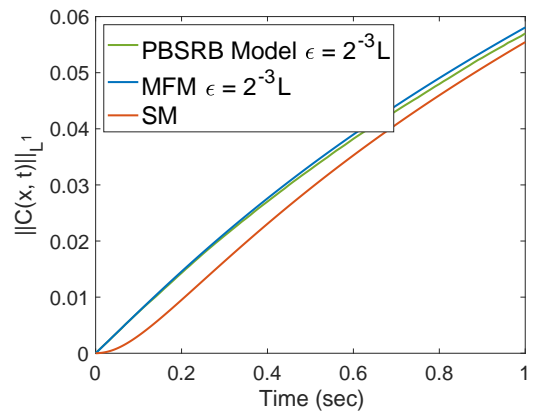

(b)

Figure 1. Evolution of the Molar Mass in one dimension estimated by 100 simulations with $\gamma=10^{4}$ and (a) reaction radius $\epsilon=2^{-7}$, (b) reaction radius $\epsilon=2^{-4} * L$.

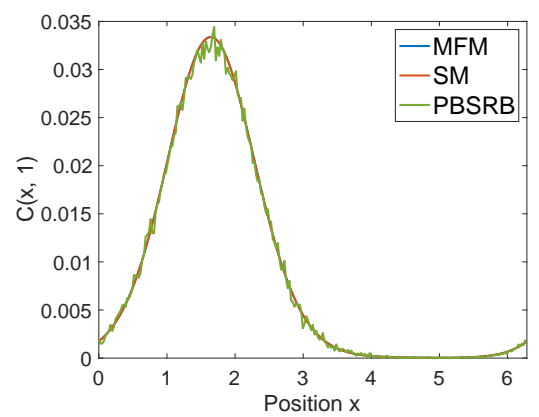

(a)

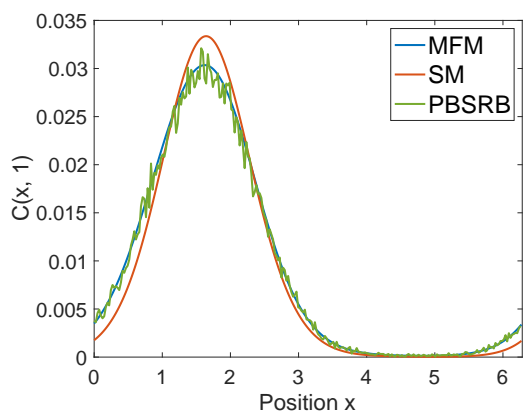

(b)

Figure 2. Spatial Molar Concentration at Time 1 in one dimension estimated by 100 simulations (a) with reaction radius $\epsilon=2^{-7}$, (b) with reaction radius $\epsilon=2^{-4} * L$.

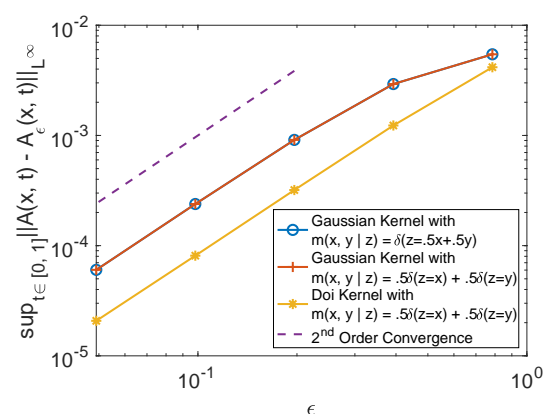

(a)

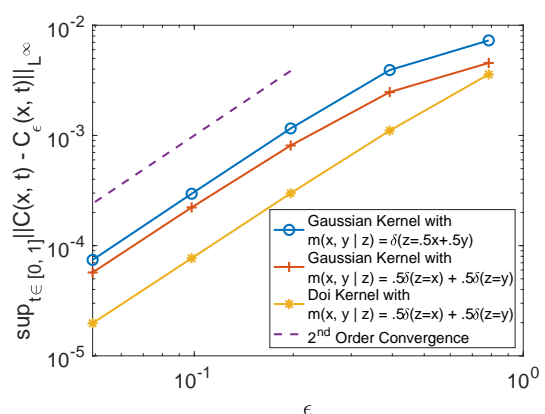

(b)

Figure 3. One Dimensional Uniform Convergence of the spatial density in the time interval $[0,1]$ (a) for species A, (b) for species C.

and placement measure $m_{1}(x, y \mid z)=.5 \delta(z-x)+.5 \delta(z-y)$. Here we fix the initial solutions for both models to be $A(x, 0)=e^{-12\left(x_{1}-1\right)^{2}-8\left(x_{2}-2\right)^{2}}, B(x, 0)=e^{-10\left(x_{1}-1\right)^{2}-5\left(x_{2}-2\right)^{2}}$ and $C(x, 0)=0$, where $x=\left(x_{1}, x_{2}\right) \in \mathbb{R}^{2}$.

Uniform second order convergence in space and time is again verified as shown in Fig. 4a. The error between the two models versus time is illustrated in Fig. 4b. We see that the maximum error over space increases to a maximum and then decreases as $t$ increases for each value of $\epsilon$. The smaller $\epsilon$ is, the smaller 


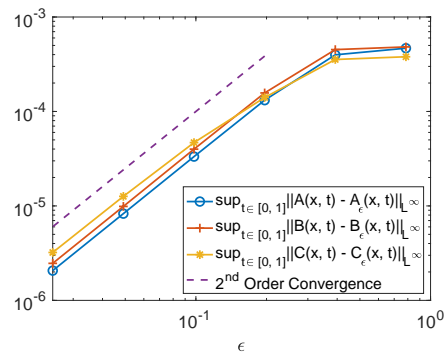

(a)

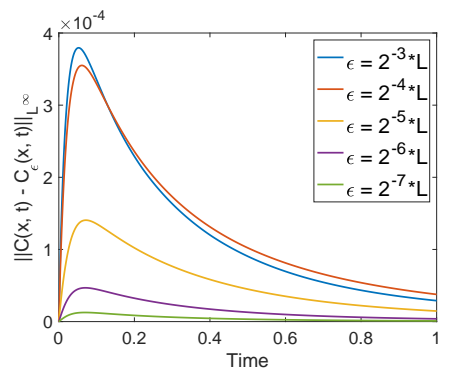

(b)

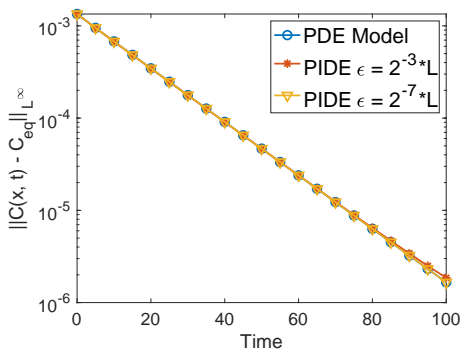

(c)

Figure 4. Two Dimensional Reversible Reactions. (a) Convergence of the spatial density uniformly in space and time interval $[0,1]$ for species A, B, and C. (b) The error between the PDE solution and the PIDE solution versus time for species C. (c) Convergence to the same constant equilibrium denoted as $C_{e q}$ for the PDE and MFM.

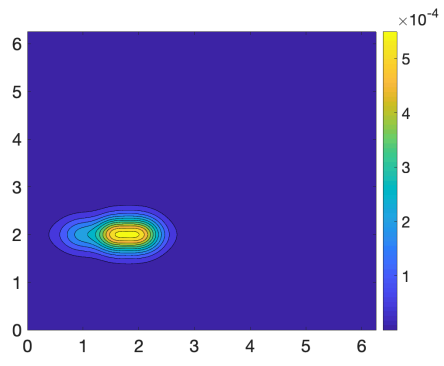

(a) SM at Time 0.1

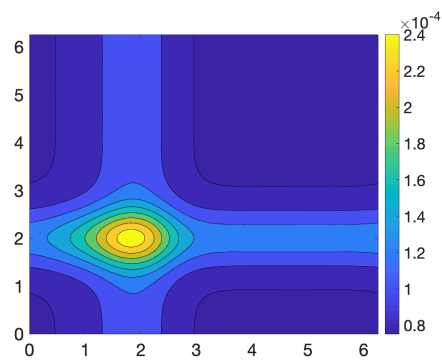

(d) SM at Time 1

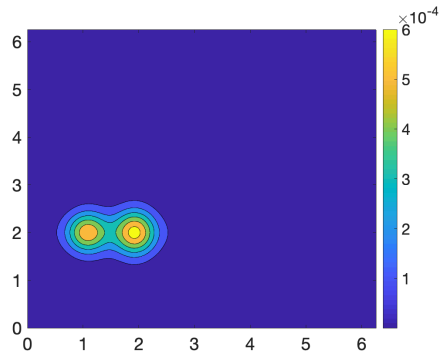

(b) MFM with $\epsilon=$ $2^{-3} * L$ at Time 0.1

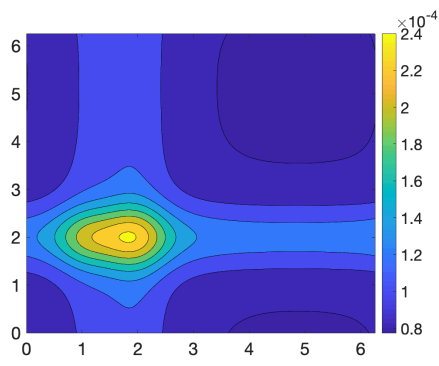

(e) MFM with $\epsilon=$ $2^{-3} * L$ at Time 1

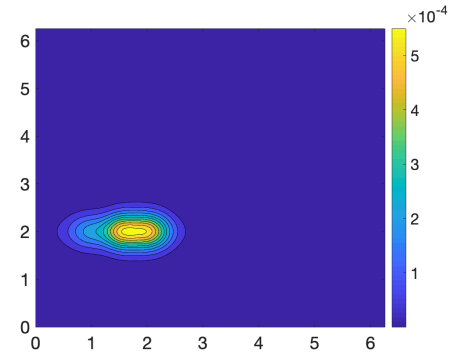

(c) MFM with $\epsilon=$ $2^{-7} * L$ at Time 0.1

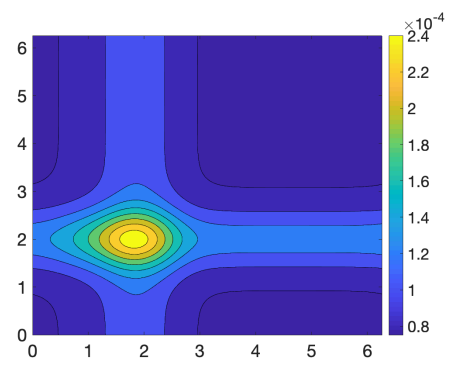

(f) MFM with $\epsilon=$ $2^{-7} * L$ at Time 1

Figure 5. Two dimensional spatial profile for species C.

the error between the solutions of the SM and the MFM. The error decreases for large times because both models converge to the same spatially uniform equilibrium solution (exponentially fast) as $t \rightarrow \infty$, as shown in Figure Fig. 4c. Here the equilibrium constant can be calculated from the corresponding mass action ODE model for the reaction using conservation laws for the reversible reaction. Let $A_{e q}, B_{e q}, C_{e q}$ denote the equilibrium concentration for species $\mathrm{A}, \mathrm{B}$ and $\mathrm{C}$ respectively in the ODE model. They satisfy $K_{\mathrm{d}} C_{e q}=A_{e q} B_{e q}$. Let $A_{0}, B_{0}, C_{0}$ denote the averaged spatial density at $t=0$ in the MFM and SM, and assume these are the initial conditions used in the ODE model. In the ODE model we have that $A_{e q}+B_{e q}+2 C_{e q}=A_{0}+B_{0}+2 C_{0}:=\operatorname{sum}$ and $B_{e q}-A_{e q}=B_{0}-A_{0}:=\operatorname{diff}$. Solving these three equations we obtain the equilibrium concentration $C_{e q}=\frac{1}{2}\left(\right.$ sum $\left.+K_{\mathrm{d}}-\sqrt{\left(\text { sum }+K_{\mathrm{d}}\right)^{2}-\left(\text { sum }^{2}-\operatorname{diff}^{2}\right)}\right)$. 
We remark that even though the dynamics of the SM and MFM are different, as long as we choose $\epsilon$ sufficiently small, in particular $\epsilon=2^{-7} L \approx .8 \% L$, there is no apparent visual difference between the two models, as illustrated in Fig. 5 .

3.3. Examples of disagreement between the PBSRD, MFM and SM models. Our previous examples illustrated our main result, exploring regimes where the MFM converges to the SM as $\epsilon \rightarrow 0$. This may suggest that one can always use the SM in applications where a deterministic model is sufficient. We now illustrate two contexts where the SM is problematic as an approximation to average concentration fields in the PBSRD model, while the MFM captures key aspects of their behavior. In Section 3.3.1 we consider a pattern formation example, the Baras-Pearson-Mansour (BPM) Model, and show the MFM is able to approximate the steady state statistics of CRDME simulations of the volume-reactivity PBSRD model. In contrast, the SM appears to converge to different steady-state statistics. In Section 3.3.2, we study a simplified version of a model for regulation of $\mathrm{T}$ cell signaling from [ZI20]. We demonstrate that the MFM is able to show the same qualitative switch-like behavior at steady state as CRDME simulations of the volume-reactivity PBSRD model in [ZI20], whereas it is not immediately clear what an appropriate SM to use for this problem would be.

3.3.1. Baras-Pearson-Mansour (BPM) Model. We consider a reaction-diffusion system with three species, U, $\mathrm{V}$ and $\mathrm{W}$ undergoing the following reactions Eq. (3.13) called the Baras-Pearson-Mansour (BPM) Model [BMP96, BPM90],

$$
U+W \stackrel{\eta_{1}}{\rightarrow} V+W, \quad 2 V \underset{\eta_{3}}{\stackrel{\eta_{2}}{\rightleftarrows}} W, \quad U \underset{\eta_{5}}{\stackrel{\eta_{4}}{\rightleftarrows}} \emptyset, \quad V \underset{\eta_{7}}{\stackrel{\eta_{6}}{\rightleftarrows}} \emptyset .
$$

We use the parameters provided in [KNBGD17] for a reaction-limited system, and fix the spatial domain as a $32 \mu m \times 32 \mu m$ square with periodic boundary conditions. The diffusivities are $D_{V}=D_{W}=D_{U} / 10=$ $0.01 \mu \mathrm{m}^{2} / \mathrm{sec}$ with rate constants $\eta_{1}=\eta_{2}=2 \times 10^{-4} \mu \mathrm{m}^{2} / \mathrm{sec}, \eta_{3}=1.0 \mathrm{sec}^{-1}, \eta_{4}=3.33 \times 10^{-3} \mathrm{sec}^{-1}$, $\eta_{5}=16.7 \mu \mathrm{m}^{-2} \mathrm{sec}^{-1}, \eta_{6}=3.67 \times 10^{-2} \mathrm{sec}^{-1}$ and $\eta_{7}=4.44 \mu \mathrm{m}^{-2} \mathrm{sec}^{-1}$. For the CRDME and MFM, we consider two bimolecular interaction length scales, $\epsilon=0.05 \mu \mathrm{m}$ and $\epsilon=0.025 \mu \mathrm{m}$ (kept the same in the two bimolecular reactions of the system). The corresponding particle-level Gaussian kernels' rates $k_{i}(i=1,2)$, for a reaction-limited system, are calibrated by imposing that

$$
\eta_{i}=\frac{1}{|\Omega|} \int_{\Omega^{2}} \frac{k_{i}}{\left(\sqrt{2 \pi \epsilon^{2}}\right)^{d}} e^{-\frac{|x-y|^{2}}{2 \epsilon^{2}}} d \boldsymbol{x} d \boldsymbol{y}
$$

where $\Omega$ denotes the spatial domain. This corresponds to matching the well-mixed reaction-rate constant in the (formal) infinite diffusivity limit. The product placement rule for the $U+W \rightarrow V+W$ reaction is to place $\mathrm{V}$ at the position of $\mathrm{U}$. The placement rule for the $2 \mathrm{~V} \rightarrow W$ reaction is to place $\mathrm{W}$ with equal probability at the position of the first $\mathrm{V}$ or the second $\mathrm{V}$. For the reverse $W \rightarrow 2 \mathrm{~V}$ reaction one $\mathrm{V}$ is placed at the position of $\mathrm{W}$, while the position of the other is determined so as to ensure detailed balance of the reversible reaction, see [IZ18].

We denote the spatial-average of the average number density fields for species $\mathrm{U}, \mathrm{V}, \mathrm{W}$ at time $t$ as $n_{U}(t), n_{V}(t), n_{W}(t)$ respectively. We initiate the system randomly around a point on the limit cycle, $\left(n_{U}(0), n_{V}(0), n_{W}(0)\right)=(1686,534,56) \mu m^{-2}$. More precisely, we generate the spatially inhomogenous initial particle number for species $s$ in voxel $V_{i}$ from a Poisson distribution with mean $n_{s}(0) \times\left|V_{i}\right|$ following [KNBGD17] and use the same initial number density for all the models considered. We use the same Fourier collocation method as in Section 3.2 to solve for the SM and MFM, choosing a time step of $d t=0.1 \mathrm{sec}$ and using $N=100$ points per coordinate axis. The CRDME is used for simulation of the underlying particle model, using the same underlying mesh.

Fig. 6 demonstrates that for all the models with $\epsilon$ sufficiently small, the short-time spatially-averaged average number densities for species $U$ agree as we proved in Theorem 3.1. At intermediate times, the stochastic reaction mechanism in the particle model facilitates faster relaxation, with smaller reactive length scales giving faster relaxation for both the CRDME and MFM. This is consistent with the observations in Figure 5 of [DYK18]. Neither the MFM or the SM give good approximations to the relaxation timescales in the CRDME simulations, though the disagreement of the SM appears less than the MFM for the two values of $\epsilon$ shown in the figure. In contrast, for very long times the MFM demonstrates better agreement with the limiting steady state value from the CRDME simulations for each value of $\epsilon$, while the SM shows a 


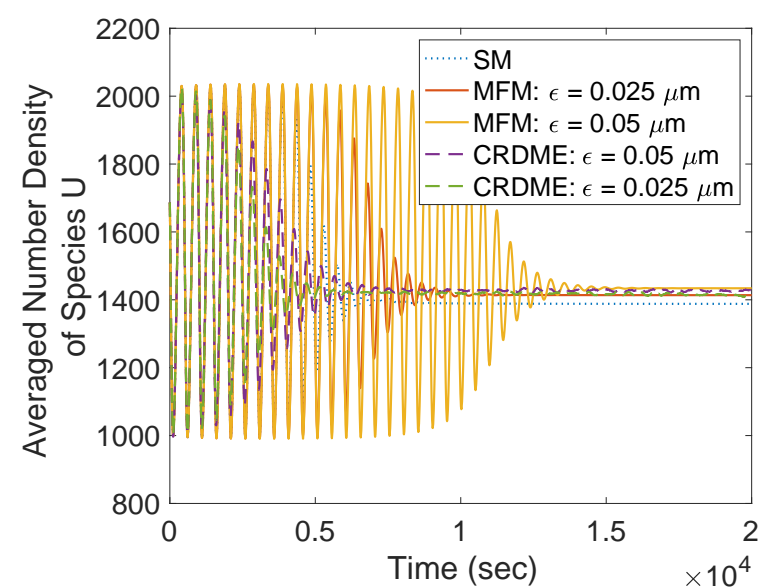

(a)

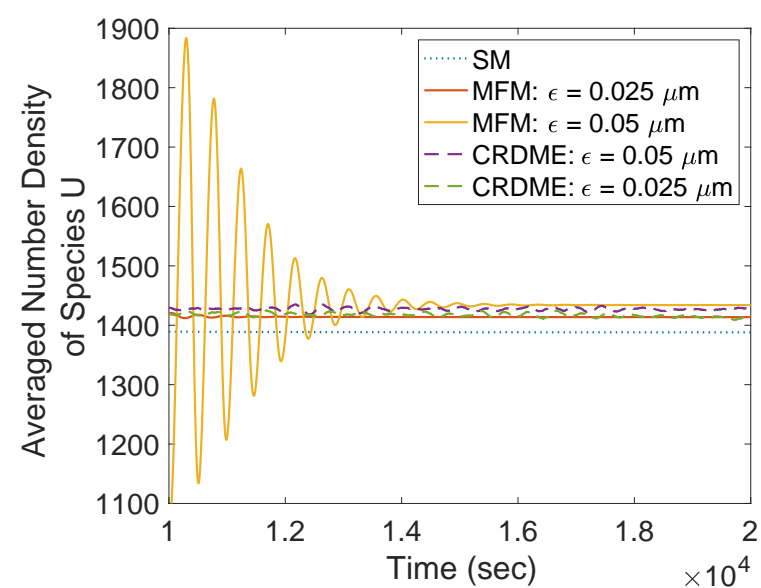

(b)

FiguRE 6. Spatially-averaged average number density of species U versus time (sec) from SM model, MFM model and CRDME model up to time $20000 \mathrm{sec}$. The results of the CRDME are averaged ovder 10 simulations. (b) is a zoomed in version of (a) focusing on the long-time behavior.

clear discrepancy (see the right panel of Fig. 6). We note that it is an open question to study how the long time behaviors of the MFM, SM and CRDME relate to each other in complex biochemical systems. We hope to explore how the bimolecular reaction range and system parameters affect the steady state pattern formations and macroscopic observables from the three models in future work.

3.3.2. Tethered Surface Receptor Interactions in T Cell Signaling. We now consider a simplified model for a tethered surface receptor interactions that occur in $\mathrm{T}$ cell signaling ( $\mathrm{T}$ cells play a key role in the adaptive immune response). The example illustrates a case where the $\mathbf{M F M}$ is well-defined, and captures key qualitative behavior of the underlying particle model, but it is not immediately clear how to formulate an appropriate SM (the assumptions of Theorem 3.1 are violated). This demonstrates that while in many contexts the SM and MFM agree well for physically-appropriate parameters, there are cases where the MFM itself serves as a useful model for biological systems.

Surface receptors within the cell membrane often have cytosolic tails that contain docking cites for cytosolic enzymes (i.e. enzymes diffusing within the cell), and regulatory sites that can be modulated by such enzymes. The length and stiffness properties of these tails then define an effective interaction distance for bimolecular reactions involving such receptors, called the molecular reach of the reaction [ZI20]. Enzymes attached to binding sites on tails interact with regulatory sites on nearby tails within the three-dimensional volume proximal to the cell membrane. In contrast, the receptors to which the tails are attached diffuse within the two-dimensional membrane surface. We therefore obtain a reaction-diffusion process of particles (receptors) moving in a two-dimensional domain but reacting through three-dimensional reaction kernels.

In [ZI20], we investigated a tethered signaling reaction in which surface membrane PD-1 proteins could inhibit activated CD-28 surface receptors, a key component in sustaining $\mathrm{T}$ cell signaling responses. We explored how the size of the molecular reach (i.e. bimolecular interaction distance) and diffusivity of the receptors could influence the efficacy of CD-28 inhibition by PD-1. Letting CD28 denote the inactivated (i.e. unphosphorylated) state, and CD28* the activated (i.e. phosphorylated) state, our model had the basic reactions that

$$
\mathrm{CD} 28 \stackrel{\lambda}{\rightarrow} \mathrm{CD}^{2} 8^{*}, \quad \mathrm{CD} 28^{*}+\mathrm{PD}-1 \stackrel{\hat{K}_{2.5 D}^{\epsilon}(\cdot) \text { or } \hat{K}_{2 D}^{\epsilon}(\cdot)}{\longrightarrow} \mathrm{CD} 28+\mathrm{PD}-1 .
$$

Here CD28 activation (phosphorylation) follows a first order reaction with rate $\lambda$. Inactivation (dephosphorylation) of CD28* is controlled by $\mathrm{PD}-1$, and modeled by a second order tethered reaction with bimolecular reaction kernel Eq. (3.14). It depends on the molecular reach $\epsilon$ derived from a polymer model for the 
cytoplasmic tail of the protein [ZI20]. The bimolecular reaction kernel is given by

$$
\hat{K}_{2.5 D}^{\epsilon}(x)=k_{2.5 D} \times\left(\frac{1}{2 \pi \epsilon^{2}}\right)^{3 / 2} \exp \left(-\frac{|x|^{2}}{2 \epsilon^{2}}\right) .
$$

Notice that Eq. (3.14) is a 3D Gaussian kernel, but the molecules will be restricted to diffuse within the twodimensional membrane surface. We therefore label it the $2.5 \mathrm{D}$ reactive kernel. To understand how having a three-dimensional interaction for particles moving in two-dimensions changes the reaction efficiacy, in [ZI20] we compared it with a purely 2D bimolecular reaction kernel. The latter is given by the 2D Gaussian kernel

$$
\hat{K}_{2 D}^{\epsilon}(x)=k_{2 D} \times\left(\frac{1}{2 \pi \epsilon^{2}}\right) \exp \left(-\frac{|x|^{2}}{2 \epsilon^{2}}\right) .
$$

We now use our MFM to study the influence of the molecular reach $\epsilon$ and diffusivity, denoted by $D$, on CD28 phosphorylation in a simplified version of the preceding model. Let us denote $A(x, t)$ as the number density of CD28 at position $x$ at time t, and $B(x, t)$ as the number density of CD28* at position $x$ at time t. For illustrative purposes, we use simplified parameters, and assume there is only one (stationary) PD-1 protein in the system. Assume the spatial domain is a $[0,50 \mathrm{~nm}] \times[0,50 \mathrm{~nm}]$ square patch of membrane, with periodic boundary conditions. The one PD-1 molecule is placed at the center of the domain, $(25,25)$, so that the number density of the PD-1 molecule is given by the constant field $\delta_{(25,25)}(x)$. The MFM for this system is then

$$
\begin{aligned}
& \frac{\partial}{\partial t} A(x, t)=D \Delta_{x} A(x, t)-\lambda A(x, t)+\hat{K}^{\epsilon}(x-(25,25)) B(x, t), \\
& \frac{\partial}{\partial t} B(x, t)=D \Delta_{x} B(x, t)+\lambda A(x, t)-\hat{K}^{\epsilon}(x-(25,25)) B(x, t),
\end{aligned}
$$

where $\hat{K}^{\epsilon}$ is $\hat{K}_{2.5 D}^{\epsilon}$ in the physiological case and $\hat{K}_{2 D}^{\epsilon}$ in the idealized case of purely two-dimensional bimolecular interactions.

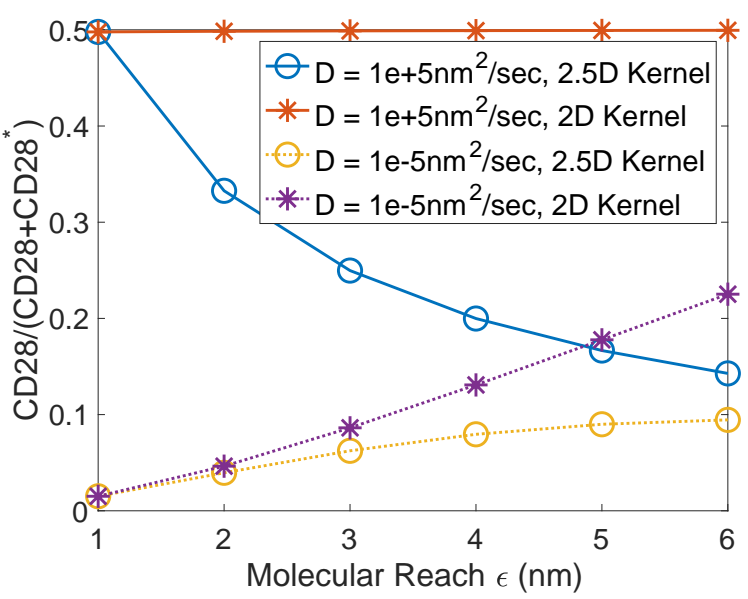

Figure 7. Steady-state fraction of CD28 that is inactivated versus molecular reach $\epsilon$ for different choices of bimolecular reaction kernel and diffusivity. Different diffusivities are labeled by different line styles: $D=10^{5} \mathrm{~nm}^{2} / \mathrm{sec}$ is a solid line and $D=10^{-5} \mathrm{~nm}^{2} / \mathrm{sec}$ is a dotted line. Different reactive kernels are labeled by different markers: the $2 \mathrm{D}$ kernel is labeled by star markers and the $2.5 \mathrm{D}$ kernel is labeled by circle markers.

In the following numerical experiments we fix $\lambda=1 \mathrm{sec}^{-1}, k_{2.5 D}=k_{2 D}=2500 \mathrm{~nm}^{2} / \mathrm{sec}$ and the initial density as constant, $A(x, 0)=10^{-4} \mathrm{~nm}^{-2}, B(x, 0)=0 \mathrm{~nm}^{-2}$. We use the same numerical methods as Section 3.2 for solving the MFM, choosing a time step of $d t=0.001 \mathrm{sec}$ and the number of spatial points per axis to be $N=100$. We study the influence of molecular reach $\epsilon$ on the steady-state fraction of CD28 in the inactivated state, illustrating the potency of PD-1. In Fig. 7 we show how this fraction is modulated 
for each reaction kernel in both the reaction-limited case (i.e. fast diffusion $D=10^{5} \mathrm{~nm}^{2} / \mathrm{sec}$, solid curve) and the diffusion-limited case (i.e. slow diffusion $D=10^{-5} \mathrm{~nm}^{2} / \mathrm{sec}$, dotted line). We see that in the case of the (unphysical) 2D kernel (star markers), in both the diffusion-limited and reaction-limited regimes the inactivated fraction is non-decreasing with respect to the reach $\epsilon$. In contrast, for the physiological 2.5D kernel (circle markers), the inactivated fraction decreases in the reaction-limited regime, but increases in the diffusion-limited regime, as the reach $\epsilon$ is increased. This behavior qualitatively reproduces what we observed for a CRDME-based particle model where both CD28 and PD-1 could diffuse in [ZI20], illustrating how the reach and diffusivity of a receptor can combine to modulate its regulatory efficacy.

While the MFM for the physiological 2.5D model follows from the underlying particle model of [ZI20], it is not immediately clear what a corresponding SM should be. One could try to just write down such a model, but it would need to capture the qualitative dependency illustrated in Fig. 7, which arises from the explicit length scale over which spatial interactions can occur in the particle model. If one instead tries to derive the $\mathbf{S M}$ as the $\epsilon \rightarrow 0$ limit of the MFM, it is also unclear what this limit should be in the $2.5 \mathrm{D}$ case, where the bimolecular reaction kernel does not satisfy the assumptions of Theorem 3.1. In particular, the kernel approaches a three-dimensional delta function, but is being used as a coefficient within a two-dimensional model. In contrast, the MFM is relatively immediate to write down, and as we have demonstrated reproduces the qualitative dependence of the fraction of inactivated receptor on the molecular reach $\epsilon$.

\section{Local Well-Posedness and Regularity Analysis}

Local existence and uniqueness can be derived from the classical contraction mapping argument, see for example [SU17]. For completeness, we present the details for the Mean Field Model (MFM) in this section. We define $\mathbb{X}=\left[C_{b, \text { unif }}\left(\mathbb{R}^{d}\right)\right]^{J}$ to be the $J$-vector space of uniformly bounded and uniformly continuous functions on $\mathbb{R}^{d}$, equipped with the norm

$$
\|\boldsymbol{\rho}\|_{\mathbb{X}}=\sup _{j=1, \cdots, J, \sup _{x \in \mathbb{R}^{d}}}\left|\rho_{j}(x)\right|,
$$

where $\boldsymbol{\rho}=\left(\rho_{1}, \rho_{2}, \cdots, \rho_{J}\right)^{T}$ and each $\rho_{j} \in C_{b, \text { unif }}\left(\mathbb{R}^{d}\right), j=1, \cdots, J$.

Applying the variation of constants formula to the MFM Eq. (2.1) we have

$$
\boldsymbol{\rho}(t)=S(t) \boldsymbol{\rho}(0)+\int_{0}^{t} S(t-s) N_{2}[\boldsymbol{\rho}](s) d s
$$

where $\boldsymbol{\rho}(t)=\left(\rho_{1}(\cdot, t), \rho_{2}(\cdot, t), \cdots, \rho_{J}(\cdot, t)\right)^{T}, S(t)$ is the heat semigroup generated by the linear diffusion operator $\operatorname{Diag}\left(D_{1} \Delta_{x}, D_{2} \Delta_{x}, \cdots, D_{J} \Delta_{x}\right)$, and $N_{2}[\boldsymbol{\rho}]=\left(\left(N_{2}[\boldsymbol{\rho}]\right)_{1},\left(N_{2}[\boldsymbol{\rho}]\right)_{2}, \cdots,\left(N_{2}[\boldsymbol{\rho}]\right)_{J}\right)^{T}$ represents the nonlinear reaction term with

$$
\begin{aligned}
\left(N_{2}[\boldsymbol{\rho}]\right)_{j}(x, t)= & -\sum_{\ell=1}^{L}\left(\frac{1}{\boldsymbol{\alpha}^{(\ell) !}} \sum_{r=1}^{\alpha_{\ell j}} \int_{\tilde{\boldsymbol{x}} \in \mathbb{X}^{(\ell)}} \delta_{x}\left(\tilde{x}_{r}^{(j)}\right) K_{\ell}(\tilde{\boldsymbol{x}})\left(\Pi_{k=1}^{J} \Pi_{s=1}^{\alpha_{\ell k}} \rho_{k}\left(\tilde{x}_{s}^{(k)}, t\right)\right) d \tilde{\boldsymbol{x}}\right) \\
& +\sum_{\ell=1}^{L}\left(\frac{1}{\boldsymbol{\alpha}^{(\ell) !}} \sum_{r=1}^{\beta_{\ell j}} \int_{\tilde{\boldsymbol{x}} \in \mathbb{X}^{(\ell)}} K_{\ell}(\tilde{\boldsymbol{x}})\left(\int_{\boldsymbol{y} \in \mathbb{Y}^{(\ell)}} \delta_{x}\left(y_{r}^{(j)}\right) m_{\ell}(\boldsymbol{y} \mid \tilde{\boldsymbol{x}}) d \boldsymbol{y}\right)\left(\Pi_{k=1}^{J} \Pi_{s=1}^{\alpha_{\ell k}} \rho_{k}\left(\tilde{x}_{s}^{(k)}, t\right)\right) d \tilde{\boldsymbol{x}}\right),
\end{aligned}
$$

for any $j=1, \cdots, J$.

Remark 4.1. $S(t)$ is a $C_{0}$-semigroup on $\mathbb{X}$ and $\|S(t) \boldsymbol{\rho}\|_{\mathbb{X}} \leq\|\boldsymbol{\rho}\|_{\mathbb{X}}$ for all $t \geq 0$ and $\boldsymbol{\rho} \in \mathbb{X}$. Furthermore, if all the diffusion coefficients are strictly positive, as we will subsequently assume, then $S(t)$ is an analytic semigroup on $\mathbb{X}$.

Fix $C_{1}>0, T_{0}$ sufficiently small, and define the Banach space $\mathbb{Y}=C\left(\left[0, T_{0}\right], \mathbb{X}\right)$ equipped with the norm

$$
\|\boldsymbol{\rho}\|_{\mathbb{Y}}=\sup _{t \in\left[0, T_{0}\right]}\|\boldsymbol{\rho}(t)\|_{\mathbb{X}}
$$

Let

$$
M=\left\{\boldsymbol{\rho} \in \mathbb{Y}:\left\|\boldsymbol{\rho}(\cdot)-S(\cdot) \boldsymbol{\rho}_{0}\right\|_{\mathbb{Y}} \leq C_{1}\right\}
$$

adapted with the same norm

$$
\|\boldsymbol{\rho}\|_{M}=\|\boldsymbol{\rho}\|_{\mathbb{Y}} .
$$


Note that by the contraction property of heat semigroup, we would have $C_{2}:=\sup _{\boldsymbol{\rho} \in M}\|\boldsymbol{\rho}\|_{M} \leq C_{1}+C_{0}$, where $C_{0}:=\left\|\rho_{0}\right\|_{M}$.

We first show $N_{2}[\boldsymbol{\rho}]$ is a smooth and locally Lipschitz function w.r.t. $\boldsymbol{\rho}$.

Lemma 4.2. Assume the reaction kernels and placement measures are of the form given in Section 2.1, with the assumed allowable types of first and second order reactions. For any $\boldsymbol{\rho}, \tilde{\boldsymbol{\rho}} \in M$ we have that the following hold:

(P1) [Boundedness]:

(P2) [Locally Lipschitz]:

$$
\left\|N_{2}[\boldsymbol{\rho}]\right\|_{M} \leq 3\left(C_{2}^{2} \vee 1\right)\left(\sum_{\ell=1}^{L} k_{\ell}\right)
$$

$$
\left\|N_{2}[\boldsymbol{\rho}]-N_{2}[\tilde{\boldsymbol{\rho}}]\right\|_{M} \leq 3\left(C_{2} \vee 1\right)\left(\sum_{\ell=1}^{L} k_{\ell}\right)\|\boldsymbol{\rho}-\tilde{\boldsymbol{\rho}}\| \|_{M},
$$

where the constants $k_{\ell}$ are defined in Assumption 2.3 and Assumption 2.5.

Proof. Proof of (P1): We have the following two estimates for the components of $N_{2}[\boldsymbol{\rho}]$.

$$
\begin{aligned}
& \left|\frac{1}{\boldsymbol{\alpha}^{(\ell) !}} \sum_{r=1}^{\alpha_{\ell j}} \int_{\tilde{\boldsymbol{x}} \in \mathbb{X}^{(\ell)}} \delta_{x}\left(\tilde{x}_{r}^{(j)}\right) K_{\ell}(\tilde{\boldsymbol{x}})\left(\Pi_{k=1}^{J} \Pi_{s=1}^{\alpha_{\ell k}} \rho_{k}\left(\tilde{x}_{s}^{(k)}, t\right)\right) d \tilde{\boldsymbol{x}}\right| \\
& \leq\left|\int_{\tilde{\boldsymbol{x}} \in \mathbb{X}^{(\ell)}} \delta_{x}\left(\tilde{x}_{1}^{(j)}\right) K_{\ell}(\tilde{\boldsymbol{x}})\left(\Pi_{k=1}^{J} \Pi_{s=1}^{\alpha_{\ell k}} \rho_{k}\left(\tilde{x}_{s}^{(k)}, t\right)\right) d \tilde{\boldsymbol{x}}\right| \\
& \leq\left|\int_{\tilde{\boldsymbol{x}} \in \mathbb{X}^{(\ell)}} \delta_{x}\left(\tilde{x}_{1}^{(j)}\right) K_{\ell}(\tilde{\boldsymbol{x}}) d \tilde{\boldsymbol{x}}\right| \times\|\boldsymbol{\rho}\|_{M}^{\left|\boldsymbol{\alpha}^{(\ell)}\right|} \leq k_{\ell}\left(C_{2}^{2} \vee 1\right),
\end{aligned}
$$

and

$$
\begin{aligned}
& \left|\frac{1}{\boldsymbol{\alpha}^{(\ell) !}} \sum_{r=1}^{\beta_{\ell j}} \int_{\tilde{\boldsymbol{x}} \in \mathbb{X}^{(\ell)}} K_{\ell}(\tilde{\boldsymbol{x}})\left(\int_{\boldsymbol{y} \in \mathbb{Y}^{(\ell)}} \delta_{x}\left(y_{r}^{(j)}\right) m_{\ell}(\boldsymbol{y} \mid \tilde{\boldsymbol{x}}) d \boldsymbol{y}\right)\left(\Pi_{k=1}^{J} \Pi_{s=1}^{\alpha_{\ell k}} \rho_{k}\left(\tilde{x}_{s}^{(k)}, t\right)\right) d \tilde{\boldsymbol{x}}\right| \\
& \leq \frac{1}{\boldsymbol{\alpha}^{(\ell) !}} \beta_{\ell j}|| \boldsymbol{\rho} \|_{M}^{\left|\boldsymbol{\alpha}^{(\ell)}\right|} \times\left|\int_{\tilde{\boldsymbol{x}} \in \mathbb{X}^{(\ell)}} K_{\ell}(\tilde{\boldsymbol{x}})\left(\int_{\boldsymbol{y} \in \mathbb{Y}^{(\ell)}} \delta_{x}\left(y_{1}^{(j)}\right) m_{\ell}(\boldsymbol{y} \mid \tilde{\boldsymbol{x}}) d \boldsymbol{y}\right) d \tilde{\boldsymbol{x}}\right| \\
& \leq 2 k_{\ell}\left(C_{2}^{2} \vee 1\right) .
\end{aligned}
$$

Then

$$
\left\|N_{2}[\boldsymbol{\rho}]\right\|_{M} \leq 3\left(C_{2}^{2} \vee 1\right)\left(\sum_{\ell=1}^{L} k_{\ell}\right) .
$$

Proof of (P2) For first and second order reactions we have

$$
\left|\Pi_{k=1}^{J} \Pi_{s=1}^{\alpha_{\ell k}} \rho_{k}\left(x_{s}^{(k)}, t\right)-\Pi_{k=1}^{J} \Pi_{s=1}^{\alpha_{\ell k}} \tilde{\rho}_{k}\left(x_{s}^{(k)}, t\right)\right| \leq\left(2|| \boldsymbol{\rho} \|\left.\right|_{M} \vee 1\right)\|\boldsymbol{\rho}-\tilde{\boldsymbol{\rho}}\|_{M} .
$$

This implies the following two estimates for the components of $N_{2}[\boldsymbol{\rho}]-N_{2}[\tilde{\boldsymbol{\rho}}]$,

$$
\begin{aligned}
& \left|\frac{1}{\boldsymbol{\alpha}^{(\ell) !}} \sum_{r=1}^{\alpha_{\ell j}} \int_{\tilde{\boldsymbol{x}} \in \mathbb{X}(\ell)} \delta_{x}\left(\tilde{x}_{r}^{(j)}\right) K_{\ell}(\tilde{\boldsymbol{x}})\left(\Pi_{k=1}^{J} \Pi_{s=1}^{\alpha_{\ell k}} \rho_{k}\left(\tilde{x}_{s}^{(k)}, t\right)-\Pi_{k=1}^{J} \Pi_{s=1}^{\alpha_{\ell k}} \tilde{\rho}_{k}\left(x_{s}^{(k)}, t\right)\right) d \tilde{\boldsymbol{x}}\right| \\
& \leq\left|\int_{\tilde{\boldsymbol{x}} \in \mathbb{X}^{(\ell)}} \delta_{x}\left(\tilde{x}_{1}^{(j)}\right) K_{\ell}(\tilde{\boldsymbol{x}})\left(\Pi_{k=1}^{J} \Pi_{s=1}^{\alpha_{\ell k}} \rho_{k}\left(\tilde{x}_{s}^{(k)}, t\right)-\Pi_{k=1}^{J} \Pi_{s=1}^{\alpha_{\ell k}} \tilde{\rho}_{k}\left(x_{s}^{(k)}, t\right)\right) d \tilde{\boldsymbol{x}}\right| \\
& \leq\left|\int_{\tilde{\boldsymbol{x}} \in \mathbb{X}(\ell)} \delta_{x}\left(\tilde{x}_{1}^{(j)}\right) K_{\ell}(\tilde{\boldsymbol{x}}) d \tilde{\boldsymbol{x}}\right| \times\left(2\|\boldsymbol{\rho}\|_{M} \vee 1\right)\|\boldsymbol{\rho}-\tilde{\boldsymbol{\rho}}\|_{M} \leq k_{\ell}\left(2 C_{2} \vee 1\right)\|\boldsymbol{\rho}-\tilde{\boldsymbol{\rho}}\|_{M},
\end{aligned}
$$

and

$$
\left|\frac{1}{\boldsymbol{\alpha}^{(\ell) !}} \sum_{r=1}^{\beta_{\ell j}} \int_{\tilde{\boldsymbol{x}} \in \mathbb{X}^{(\ell)}} K_{\ell}(\tilde{\boldsymbol{x}})\left(\int_{\boldsymbol{y} \in \mathbb{Y}^{(\ell)}} \delta_{x}\left(y_{r}^{(j)}\right) m_{\ell}(\boldsymbol{y} \mid \tilde{\boldsymbol{x}}) d \boldsymbol{y}\right)\left(\Pi_{k=1}^{J} \Pi_{s=1}^{\alpha_{\ell k}} \rho_{k}\left(\tilde{x}_{s}^{(k)}, t\right)-\Pi_{k=1}^{J} \Pi_{s=1}^{\alpha_{\ell k}} \tilde{\rho}_{k}\left(x_{s}^{(k)}, t\right)\right) d \tilde{\boldsymbol{x}}\right|
$$




$$
\begin{aligned}
& \leq \frac{1}{\boldsymbol{\alpha}^{(\ell) !}} \beta_{\ell j}\left(2\|\boldsymbol{\rho}\|_{M} \vee 1\right)\|\boldsymbol{\rho}-\tilde{\boldsymbol{\rho}}\|_{M} \times\left|\int_{\tilde{\boldsymbol{x}} \in \mathbb{X}^{(\ell)}} K_{\ell}(\tilde{\boldsymbol{x}})\left(\int_{\boldsymbol{y} \in \mathbb{Y}^{(\ell)}} \delta_{x}\left(y_{1}^{(j)}\right) m_{\ell}(\boldsymbol{y} \mid \tilde{\boldsymbol{x}}) d \boldsymbol{y}\right) d \tilde{\boldsymbol{x}}\right| \\
& \leq 2 k_{\ell}\left(2 C_{2} \vee 1\right)\|\boldsymbol{\rho}-\tilde{\boldsymbol{\rho}}\|_{M} .
\end{aligned}
$$

Therefore,as claimed, we obtain that

$$
\left\|N_{2}[\boldsymbol{\rho}]-N_{2}[\tilde{\boldsymbol{\rho}}]\right\|\left\|_{M} \leq 3\left(\sum_{k=1, \cdots, L} k_{\ell}\right)\left(2 C_{2} \vee 1\right)\right\| \boldsymbol{\rho}-\tilde{\boldsymbol{\rho}}\|\|_{M} .
$$

Making use of the boundedness and locally Lipschitz properties of $N_{2}[\cdot]$ from Lemma 4.2, we obtain

Theorem 4.3. Assume the conditions of Lemma 4.2. For all $C_{0}>0$, there exists a $T_{0}>0$ such that for $\boldsymbol{\rho}_{0} \in \mathbb{X}$, with $\left\|\boldsymbol{\rho}_{0}\right\|_{\mathbb{X}} \leq C_{0}$, there exists a unique mild solution $\boldsymbol{\rho} \in C\left(\left[0, T_{0}\right], \mathbb{X}\right)$ to Eq. (2.1) with $\boldsymbol{\rho}(0)=\boldsymbol{\rho}_{0}$.

Proof. Let $F[\boldsymbol{\rho}]$ be the righthand side of the variation of constant formula Eq. (3.1). We'll show in the following that $F$ maps $M$ to $M$ and is a contraction mapping in $M$ using the contraction property of heat semigroup and the properties of the reaction operator in Lemma 4.2.

(1) $F$ maps $M$ to $M$ follows from

$$
\begin{aligned}
\left\|F[\boldsymbol{\rho}]-S(t) \boldsymbol{\rho}_{0}\right\|_{M} & =\sup _{t \in\left[0, T_{0}\right]}\left\|F[\boldsymbol{\rho}](t)-S(t) \boldsymbol{\rho}_{0}\right\|_{\mathbb{X}}=\sup _{t \in\left[0, T_{0}\right]}\left\|\int_{0}^{t} S(t-s) N_{2}[\boldsymbol{\rho}](s) d s\right\|_{\mathbb{X}} \\
& \leq \sup _{t \in\left[0, T_{0}\right]} \int_{0}^{t}\left\|S(t-s) N_{2}[\boldsymbol{\rho}](s)\right\|_{\mathbb{X}} d s \leq \sup _{t \in\left[0, T_{0}\right]} \int_{0}^{t}\left\|N_{2}[\boldsymbol{\rho}](s)\right\|_{\mathbb{X}} d s \\
& \leq T_{0}\left(3\left(C_{2}^{2} \vee 1\right)\left(\sum_{\ell=1}^{L} \kappa_{\ell}\right)\right) \leq C_{1},
\end{aligned}
$$

as long as $T_{0} \leq \frac{C_{1}}{3\left(C_{2}^{2} \vee 1\right)\left(\sum_{\ell=1}^{L} \kappa_{\ell}\right)}$.

(2) $F$ is a contraction mapping in $M$ follows from

$$
\begin{aligned}
\|F[\boldsymbol{\rho}]-F[\tilde{\boldsymbol{\rho}}]\|_{M} & =\sup _{t \in\left[0, T_{0}\right]}\|F[\boldsymbol{\rho}](t)-F[\tilde{\boldsymbol{\rho}}]\|_{\mathbb{X}} \leq \sup _{t \in\left[0, T_{0}\right]} \int_{0}^{t}\left\|S(t-s)\left(N_{2}[\boldsymbol{\rho}](s)-N_{2}[\tilde{\boldsymbol{\rho}}](s)\right)\right\|_{\mathbb{X}} d s \\
& \leq \sup _{t \in\left[0, T_{0}\right]} \int_{0}^{t}\left\|N_{2}[\boldsymbol{\rho}](s)-N_{2}[\tilde{\boldsymbol{\rho}}](s)\right\|_{\mathbb{X}} d s \leq \sup _{t \in\left[0, T_{0}\right]} \int_{0}^{t}\left\|N_{2}[\boldsymbol{\rho}]-N_{2}[\tilde{\boldsymbol{\rho}}]\right\|_{M} d s \\
& \leq T_{0}\left(3\left(2 C_{2} \vee 1\right)\left(\sum_{\ell=1}^{L} \kappa_{\ell}\right)\right)\|\boldsymbol{\rho}(s)-\tilde{\boldsymbol{\rho}}(s)\|_{M} \leq \frac{1}{2}\|\boldsymbol{\rho}(s)-\tilde{\boldsymbol{\rho}}(s)\|_{M},
\end{aligned}
$$

as long as $T_{0} \leq \frac{1}{6\left(2 C_{2} \vee 1\right)\left(\sum_{\ell=1}^{L} \kappa_{\ell}\right)}$.

Therefore, by the contraction mapping theorem there exists a unique mild solution $\boldsymbol{\rho} \in C\left(\left[0, T_{0}\right], \mathbb{X}\right)$ to Eq. (2.1) satisfying Eq. (3.1).

Theorem 4.4. Under the conditions of Theorem 4.3, the mild solution $\rho_{j}(x, t) \in C\left(\left[0, T_{0}\right], C_{b, u n i f}\left(\mathbb{R}^{d}\right)\right) \cap$ $C^{1}\left(\left(0, T_{0}\right], C^{2}\left(\mathbb{R}^{d}\right)\right)$ is a classical solution to Eq. (2.1). Furthermore, if $\rho_{j}(x, 0) \in C_{b}^{1}\left(\mathbb{R}^{d}\right)$, for all $j=1, \cdots, J$, then $\rho_{j}(x, t) \in C_{b}^{2}\left(\mathbb{R}^{d}\right)$ for any $t \in\left(0, T_{0}\right]$.

Proof. Since $\boldsymbol{\rho} \in C\left(\left[0, T_{0}\right], \mathbb{X}\right)$ as we showed in Theorem 4.3, classical results for nonhomogenous Cauchy problems give that $\rho_{j}(x, t) \in C^{1}\left(\left(0, T_{0}\right], C^{2}\left(\mathbb{R}^{d}\right)\right.$ ) (see Chapter 2.3 Theorem 7 in [E10]). $\boldsymbol{\rho}$ is hence a classical solution. We'll next show that under the condition $\rho_{j}(x, 0) \in C_{b}^{1}\left(\mathbb{R}^{d}\right)$ then $\rho_{j}(x, t) \in C_{b}^{2}\left(\mathbb{R}^{d}\right)$ for $t \in\left(0, T_{0}\right]$, i.e. all the first and second partial derivatives in $x$ are bounded for $0<t \leq T_{0}$.

Let us denote $\Phi(x, t)$ as the fundamental solution of $d$-dimensional heat equation. Note that for any $x \in \mathbb{R}^{d}$

$$
\int_{\mathbb{R}^{d}}\left|\partial_{x_{i}} \Phi(x-y, t)\right| d y=\sqrt{\frac{1}{\pi t}} .
$$


We establish two estimates for the first and second partial derivatives for $\rho_{j}(x, t), t \in\left(0, T_{0}\right]$. We claim

(Inequality 1)

$$
\left\|\partial_{x_{i}} \rho_{j}(x, t)\right\|_{C_{b}\left(\mathbb{R}^{d}\right)} \leq\left\|\rho_{j}(x, 0)\right\|_{C_{b}^{1}\left(\mathbb{R}^{d}\right)}+6\left(C_{2}^{2} \vee 1\right)\left(\sum_{\ell=1}^{L} k_{\ell}\right) \sqrt{\frac{T_{0}}{\pi}}
$$

for any $0<t \leq T_{0}$, any $i=1, \cdots, d$, and $j=1, \cdots, J$. Starting from Eq. (3.1), we have that

$$
\begin{aligned}
\left|\partial_{x_{i}} \rho_{j}(x, t)\right| & =\left|\partial_{x_{i}} \int_{\mathbb{R}^{d}} \Phi(x-y, t) \rho_{j}(y, 0) d y+\partial_{x_{i}} \int_{0}^{t} \int_{\mathbb{R}^{d}} \Phi(x-y, t-s)\left(N_{2}[\boldsymbol{\rho}]\right)_{j}(y, s) d y d s\right| \\
& \leq \int_{\mathbb{R}^{d}}\left|\Phi ( x - y , t ) \left\|\partial_{x_{i}} \rho_{j}(y, 0)\left|d y+\left\|N_{2}[\boldsymbol{\rho}]\right\|_{M} \times \int_{0}^{t} \int_{\mathbb{R}^{d}}\right| \partial_{x_{i}} \Phi(x-y, t-s) \mid d y d s\right.\right. \\
& =\left\|\rho_{j}(x, 0)\right\|_{C_{b}^{1}\left(\mathbb{R}^{d}\right)}+\left\|N_{2}[\boldsymbol{\rho}]\right\|_{M} \times \int_{0}^{t} \sqrt{\frac{1}{\pi(t-s)}} d s \\
& \leq\left\|\rho_{j}(x, 0)\right\|_{C_{b}^{1}\left(\mathbb{R}^{d}\right)}+3\left(C_{2}^{2} \vee 1\right)\left(\sum_{\ell=1}^{L} k_{\ell}\right) \times 2 \sqrt{\frac{t}{\pi}},
\end{aligned}
$$

where in the last inequality, we use the estimates for $\left\|N_{2}[\boldsymbol{\rho}]\right\|_{M}$ in Lemma 4.2 and recall that we denote $C_{2}:=\sup _{\rho \in M}\|\rho\|_{M}$.

For the second derivatives, we claim

(Inequality 2) $\quad\left\|\partial_{x_{i}, x_{k}} \rho_{j}(x, t)\right\|_{C_{b}\left(\mathbb{R}^{d}\right)} \leq\left\|\rho_{j}(x, 0)\right\|_{C_{b}^{1}\left(\mathbb{R}^{d}\right)} \sqrt{\frac{1}{\pi t}}+6 C_{4}\left(2 C_{2} \vee 1\right)\left(\sum_{\ell=1}^{L} k_{\ell}\right) \sqrt{\frac{T_{0}}{\pi}}$

for any $0<t \leq T_{0}$, any $i, k=1, \cdots, d$, and $j=1, \cdots, J$, where we have denoted $C_{4}=\left.\sup _{j=1, \cdots, J}\left\|\rho_{j}(x, 0)\right\|\right|_{C_{b}^{1}\left(\mathbb{R}^{d}\right)}+$ $6\left(C_{2}^{2} \vee 1\right)\left(\sum_{\ell=1}^{L} k_{\ell}\right) \sqrt{\frac{T_{0}}{\pi}}$. Using that $\left\|\partial_{x_{i}} \rho_{j}(x, t)\right\|_{C_{b}\left(\mathbb{R}^{d}\right)} \leq C_{4}$ from (Inequality $\mathbf{1}$ ) we have

$$
\begin{aligned}
& \left|\partial_{x_{i}}\left(N_{2}[\boldsymbol{\rho}]\right)_{j}(x, t)\right| \\
& =-\sum_{\ell=1}^{L}\left(\frac{1}{\boldsymbol{\alpha}^{(\ell) !}} \sum_{r=1}^{\alpha_{\ell j}} \partial_{x_{i}} \int_{\tilde{\boldsymbol{x}} \in \mathbb{X}^{(\ell)}} \delta_{x}\left(\tilde{x}_{r}^{(j)}\right) K_{\ell}(\tilde{\boldsymbol{x}})\left(\Pi_{k=1}^{J} \Pi_{s=1}^{\alpha_{\ell k}} \rho_{k}\left(\tilde{x}_{s}^{(k)}, t\right)\right) d \tilde{\boldsymbol{x}}\right) \\
& +\sum_{\ell=1}^{L}\left(\frac{1}{\boldsymbol{\alpha}^{(\ell) !}} \sum_{r=1}^{\beta_{\ell j}} \partial_{x_{i}} \int_{\tilde{\boldsymbol{x}} \in \mathbb{X}(\ell)} K_{\ell}(\tilde{\boldsymbol{x}})\left(\int_{\boldsymbol{y} \in \mathbb{Y}^{(\ell)}} \delta_{x}\left(y_{r}^{(j)}\right) m_{\ell}(\boldsymbol{y} \mid \tilde{\boldsymbol{x}}) d \boldsymbol{y}\right)\left(\Pi_{k=1}^{J} \Pi_{s=1}^{\alpha_{\ell k}} \rho_{k}\left(\tilde{x}_{s}^{(k)}, t\right)\right) d \tilde{\boldsymbol{x}}\right) \\
& \leq \sum_{\ell=1}^{L} \frac{\beta_{\ell j}+\alpha_{\ell j}}{\boldsymbol{\alpha}^{(\ell) !}} \times k_{\ell}\left(2 C_{2} \vee 1\right) \sup _{k=1, \cdots, J}\left\|\partial_{x_{m}} \rho_{k}(x, t)\right\|_{C_{b}\left(\mathbb{R}^{d}\right)} \quad \text { (by Lemma B.1-Lemma B.2) } \\
& \leq 3 C_{4}\left(2 C_{2} \vee 1\right)\left(\sum_{\ell=1}^{L} k_{\ell}\right) \text {. }
\end{aligned}
$$

Using Eq. (3.1)

$$
\begin{aligned}
\left|\partial_{x_{i}, x_{k}} \rho_{j}(x, t)\right| & =\left|\partial_{x_{i}, x_{k}} \int_{\mathbb{R}^{d}} \Phi(x-y, t) \rho_{j}(y, 0) d y+\partial_{x_{i}, x_{k}} \int_{0}^{t} \int_{\mathbb{R}^{d}} \Phi(x-y, t-s)\left(N_{2}[\boldsymbol{\rho}]\right)_{j}(y, s) d y d s\right| \\
\leq & \int_{\mathbb{R}^{d}}\left|\partial_{x_{i}} \Phi(x-y, t)\left\|\partial_{x_{k}} \rho_{j}(y, 0) \mid d y+\sup _{t \in\left(0, T_{0}\right]}\right\| \partial_{x_{i}}\left(N_{2}[\boldsymbol{\rho}]\right)_{j}(x, t) \|_{C_{b}\left(\mathbb{R}^{d}\right)}\right. \\
& \times \int_{0}^{t} \int_{\mathbb{R}^{d}}\left|\partial_{x_{k}} \Phi(x-y, t-s)\right| d y d s \\
\leq & \left\|\rho_{j}(x, 0)\right\|_{C_{b}^{1}\left(\mathbb{R}^{d}\right)} \sqrt{\frac{1}{\pi t}}+3 C_{4}\left(2 C_{2} \vee 1\right)\left(\sum_{\ell=1}^{L} \kappa_{\ell}\right) \times \int_{0}^{t} \sqrt{\frac{1}{\pi(t-s)}} d s \\
\leq & \left\|\rho_{j}(x, 0)\right\|_{C_{b}^{1}\left(\mathbb{R}^{d}\right)} \sqrt{\frac{1}{\pi t}}+3 C_{4}\left(2 C_{2} \vee 1\right)\left(\sum_{\ell=1}^{L} \kappa_{\ell}\right) \times 2 \sqrt{\frac{t}{\pi}}
\end{aligned}
$$


Thus we conclude $\rho_{j}(x, t) \in C_{b}^{2}\left(\mathbb{R}^{d}\right)$ for any $t \in\left(0, T_{0}\right]$ as claimed and the theorem has been proven.

For the Standard Model $(\mathbf{S M})$, let $N_{1}[\boldsymbol{\rho}]=\left(\left(N_{1}[\boldsymbol{\rho}]\right)_{1},\left(N_{1}[\boldsymbol{\rho}]\right)_{2}, \cdots,\left(N_{1}[\boldsymbol{\rho}]\right)_{J}\right)^{T}$ represent the nonlinear reaction term with

$$
\left(N_{1}[\boldsymbol{\rho}]\right)_{j}=\sum_{\ell=1}^{L} \kappa_{\ell}\left(\beta_{\ell j}-\alpha_{\ell j}\right)\left(\Pi_{k=1}^{J} \rho_{k}(x, t)^{\alpha_{\ell k}}\right),
$$

for $1 \leq j \leq J$. It is standard to show that $N_{1}[\boldsymbol{\rho}]$ is a smooth and locally Lipschitz function w.r.t. $\boldsymbol{\rho}$. Analogously to the previous calculations for the MFM, we can obtain

Theorem 4.5. For all $C_{0}>0$, there exists a $T_{0}>0$ such that for $\boldsymbol{\rho}_{0} \in \mathbb{X}$, with $\left\|\boldsymbol{\rho}_{0}\right\|_{\mathbb{X}} \leq C_{0}$, there exists a unique mild solution $\boldsymbol{\rho} \in C\left(\left[0, T_{0}\right], \mathbb{X}\right)$ to Eq. (2.2) with $\boldsymbol{\rho}(0)=\boldsymbol{\rho}_{0}$.

Theorem 4.6. Under the condition of Theorem 4.5, then the mild solution $\rho_{j}(x, t) \in C\left(\left[0, T_{0}\right], C_{b, \text { unif }}\left(\mathbb{R}^{d}\right)\right) \cap$ $C^{1}\left(\left(0, T_{0}\right], C^{2}\left(\mathbb{R}^{d}\right)\right)$ is a classical solution to Eq. (2.2). Furthermore when assuming $\rho_{j}(x, 0) \in C_{b}^{1}\left(\mathbb{R}^{d}\right)$, for all $j=1, \cdots, J$, indeed $\rho_{j}(x, t) \in C_{b}^{2}\left(\mathbb{R}^{d}\right)$ for any $t \in\left(0, T_{0}\right]$.

\section{On Global Well-Posedness}

Global existence in time of the classical solution to the SM Eq. (2.2) for general reaction systems is a difficult open problem. We refer the interested reader to the recent review article [P10] for survey of the current state of the art. The recent papers by [FMT20, CGV19, S18] also deal with global well-posedness of reaction-diffusion systems under various combinations of growth and mass control assumptions.

For two main reasons, the setup of this paper is only partially covered by the existing literature. First, we are dealing with non-local systems of equations (the MFM) while the vast majority of the literature has concentrated on local systems of equations (such as the $\mathbf{S M}$ ). Second, since our main interest in this paper is to examine conditions under which the $\mathbf{S M}$ is a special case of the MFM, we need to be able to assume that the reaction kernels converge to delta Dirac masses, see Assumption 2.3, which would then require uniform bounds with respect to this approximation (see Theorem 3.1). The latter precludes us from being able to work with global boundedness assumptions on the reaction kernels (see also Remark 5.2 for a more detailed explanation of this).

In this section we demonstrate that global well-posedness can be proven to hold for our non-local MFM Eq. (2.1) in, at least, the cases of $A+B \rightleftarrows C+D$ and $A+B \rightleftarrows C$ (the latter under specific choices for the placement measures). The case of $A+B \rightleftarrows C+D$ can be addressed using the results of [FMT20], using the mass conservation property of the non-local system, see Lemma 5.1. This approach, however, fails for the non-local $A+B \rightleftarrows C$ reaction. We address the non-local $A+B \rightleftarrows C$ reaction in Lemma 5.3 by modifying an argument of [P10] to deal with the non-local nature of the equations, which uses in an essential way that two of the equations have only linear growth. We stress here that the two methods are different in nature; the first is based on mass conservation properties, while the second is based on finding a linear combination of the equations with linear growth.

It is an interesting open problem to address global well-posedness in a more unifying way generally for the MFM Eq. (2.1). However, this is outside the scope of this paper, whose primary focus is elucidating how the commonly used SM approximates the rigorous large population limit of PBSRD systems given by the MFM.

We begin in Lemma 5.1 by addressing the $A+B \rightleftarrows C+D$ reaction network. In Remark 5.2 we make a number of comments on alternative approaches in the literature that might be used to establish global existence.

Lemma 5.1. For the $A+B \rightleftarrows C+D$ reaction, both the $\boldsymbol{S M}$ and the $\boldsymbol{M F M}$ are globally well posed, i.e. Theorem 4.6 and Theorem 4.4 hold for all $T_{0}<\infty$.

Proof. By the results of [FMT20], global well-posedness will follow if the following additional conditions hold

(A1) Local Lipschitz and Preservation of Positivity: For all $j=1, \cdots, J,(N[\rho])_{j}$ is locally Lipschitz and $(N[\boldsymbol{\rho}])_{j} \geq 0$ for all $\boldsymbol{\rho} \geq 0$ with $\rho_{j}=0$,

(A2) Mass Control: $\sum_{j=1}^{J} w_{j} \times(N[\boldsymbol{\rho}])_{j} \leq C_{0}+C_{1} \sum_{j=1}^{J} \rho_{j}$, for $\boldsymbol{\rho} \geq 0$, some constants $C_{0}$ and $C_{1}$, and some set of $\left\{w_{j}\right\}_{j=1, \cdots, J}$ with $w_{j}>0$, 
(A3) (Super)-Quadratic Growth: $\left|(N[\boldsymbol{\rho}])_{j}\right| \leq C\left(1+\|\boldsymbol{\rho}\|^{2+\epsilon}\right)$, for $\boldsymbol{\rho} \geq 0$, some constant $C, \epsilon>0$ and all $j$,

where $N[\boldsymbol{\rho}]$ represents the nonlinear reaction term of a general reaction-diffusion equation, including our SM and MFM. Here, in condition (A3), $\|\cdot\|$ represents the uniform norm in space, i.e. $\|\boldsymbol{\rho}\|=\sup _{j=1, \cdots, J} \sup _{x \in \mathbb{R}^{d}}\left|\rho_{j}(x)\right|$. This is slightly different from what is assumed in [FMT20], but an examination of the proofs in [FMT20] shows that the argument goes through in this norm.

Let us denote by $\rho_{1}(x, t), \cdots, \rho_{4}(x, t)$ the concentration at position $x$ and time $t$ for species $\mathrm{A}, \mathrm{B}, \mathrm{C}, \mathrm{D}$ respectively. Let $\boldsymbol{\rho}=\left(\rho_{1}, \rho_{2}, \rho_{3}, \rho_{4}\right)^{T}$. Without loss of generality, we may assume that once the forward reaction happens, $\mathrm{A}$ becomes $\mathrm{C}$ and $\mathrm{B}$ becomes $\mathrm{D}$, whereas once the backward reaction happens, $\mathrm{C}$ becomes A and D becomes B. Then the reaction term $N[\boldsymbol{\rho}]=\left((N[\boldsymbol{\rho}])_{1},(N[\boldsymbol{\rho}])_{2},(N[\boldsymbol{\rho}])_{3},(N[\boldsymbol{\rho}])_{4}\right)^{T}$ for the MFM Model is

$$
N[\boldsymbol{\rho}](x, t)=\left(\begin{array}{c}
-\left(\int_{\mathbb{R}^{d}} K_{1}(x, y) \rho_{2}(y, t) d y\right) \rho_{1}(x, t)+\left(\int_{\mathbb{R}^{d}} K_{2}(x, w) \rho_{4}(w, t) d w\right) \rho_{3}(x, t) \\
-\left(\int_{\mathbb{R}^{d}} K_{1}(y, x) \rho_{1}(y, t) d y\right) \rho_{2}(x, t)+\left(\int_{\mathbb{R}^{d}} K_{2}(z, x) \rho_{3}(z, t) d w\right) \rho_{4}(x, t) \\
+\left(\int_{\mathbb{R}^{d}} K_{1}(x, y) \rho_{2}(y, t) d y\right) \rho_{1}(x, t)-\left(\int_{\mathbb{R}^{d}} K_{2}(x, w) \rho_{4}(w, t) d w\right) \rho_{3}(x, t) \\
+\left(\int_{\mathbb{R}^{d}} K_{1}(y, x) \rho_{1}(y, t) d y\right) \rho_{2}(x, t)-\left(\int_{\mathbb{R}^{d}} K_{2}(z, x) \rho_{3}(z, t) d w\right) \rho_{4}(x, t)
\end{array}\right)
$$

Preservation of positivity is satisfied by the non-negativity of the reaction kernel $K_{1}(x, y)$ and $K_{2}(z, w)$. A local Lipschitz condition is shown in Lemma 4.2, while $\sum_{j=1}^{4}(N[\boldsymbol{\rho}])_{j}=0$ gives (A2). By symmetry, it is sufficient to show (A3) when $j=1$.

$$
\begin{aligned}
\left|(N[\boldsymbol{\rho}])_{1}\right| & =\left|-\left(\int_{\mathbb{R}^{d}} K_{1}(x, y) \rho_{2}(y, t) d y\right) \rho_{1}(x, t)+\left(\int_{\mathbb{R}^{d}} K_{2}(x, w) \rho_{4}(w, t) d w\right) \rho_{3}(x, t)\right|, \\
& \leq\left(\int_{\mathbb{R}^{d}} \hat{K}_{1}(x-y)\left|\rho_{2}(y, t)\right| d y\right)\left|\rho_{1}(x, t)\right|+\left(\int_{\mathbb{R}^{d}} \hat{K}_{2}(x-w)\left|\rho_{4}(w, t)\right| d w\right)\left|\rho_{3}(x, t)\right|, \\
& \leq\left(\left\|\hat{K}_{1}\right\|_{L^{1}}+\left\|\hat{K}_{2}\right\|_{L^{1}}\right)\|\boldsymbol{\rho}\|^{2} .
\end{aligned}
$$

Thus, conditions (A1)-(A3) are satisfied for the MFM Model. Note, all the constants involved do not depend on $\epsilon$. The same argument works for the SM model by choosing the reaction kernels to be delta functions.

Remark 5.2. For completeness we mention here that apart from [FMT20], [CGV19] and [S18] have also addressed the global well-posedness question of general reaction-diffusion systems with condition (A1), (A2), and (A3) with $C_{1}=0$ plus an additional entropy condition.

In addition, in principle, one could also use the regularized effects of the bimolecular reaction kernel in order to prove well-posedness of the $A+B \rightleftarrows C+D$ system, as in Section 7 in [LLN19]. However, this method of proof requires boundedness of the reaction kernel whereas for the $\boldsymbol{S M}$, the reaction kernel term is essentially a Dirac delta function, which is rather singular. The idea of the method of proof in [LLN19] is that under the conditions (A1), (A2) and (A3), $\left\|\rho_{j}(x, t)\right\|_{L^{1}\left(\mathbb{R}^{d}\right)}$ is bounded for any time $t \in[0, \infty)$ for all $j=1, \cdots, J$. Then the main argument of [LLN19] is that by the prior $L^{1}$ bound on the solutions and the $L^{\infty}$ bound on the reaction kernels, the regularized (convoluted) quadratic growth term can be bounded by linear growth of the solutions, and thereby admits global existence. However, as $\epsilon \rightarrow 0$, the $L^{\infty}$ bound of the reaction kernel unfortunately is not uniform in the approximation parameter $\epsilon$. Therefore, given that our aim is to compare the two models, this method of proof is not immediately applicable to our case.

As such, we found that for our problem of interest using [FMT20] was more straight-forward for the non-local $A+B \rightleftarrows C+D$ system.

We now address global existence for the $A+B \rightleftarrows C$ reaction network.

Lemma 5.3. For the case of the $A+B \rightleftarrows C$ reaction, by choosing the binding placement measure $m_{1}(z \mid x, y)=$ $p \delta(z-x)+(1-p) \delta(z-y)$, for some $p \in[0,1]$ and assuming the detailed balance condition Eq. (3.10) in the $\boldsymbol{M F M}$, solutions to both the $\boldsymbol{S M}$ and the $\boldsymbol{M F M}$ exist globally, i.e. Theorem 4.6 and Theorem 4.4 hold for all $T_{0}<\infty$. 
Proof. We only address the MFM model as the situation for the local model SM is immediately covered by the results of both [P10] and [FMT20].

Let us denote by $\rho_{1}(x, t), \cdots, \rho_{3}(x, t)$ the concentration at position $x$ and time $t$ for species $\mathrm{A}, \mathrm{B}, \mathrm{C}$ respectively. Let $T$ be the maximal existence time for $L^{\infty}$ solutions. The MFM Model is

$$
\begin{aligned}
& \partial_{t} \rho_{1}(x, t)=D^{\mathrm{A}} \Delta \rho_{1}(x, t)-\left(\int_{\mathbb{R}^{d}} K_{1}(x, y) \rho_{2}(y, t) d y\right) \rho_{1}(x, t)+\int_{\mathbb{R}^{d}} K_{2}(z)\left(\int_{\mathbb{R}^{d}} m_{2}(x, y \mid z) d y\right) \rho_{3}(z, t) d z \\
& \partial_{t} \rho_{2}(y, t)=D^{\mathrm{B}} \Delta \rho_{2}(y, t)-\left(\int_{\mathbb{R}^{d}} K_{1}(x, y) \rho_{1}(x, t) d x\right) \rho_{2}(y, t)+\int_{\mathbb{R}^{d}} K_{2}(z)\left(\int_{\mathbb{R}^{d}} m_{2}(x, y \mid z) d x\right) \rho_{3}(z, t) d z \\
& \partial_{t} \rho_{3}(z, t)=D^{\mathrm{C}} \Delta \rho_{3}(z, t)-K_{2}(z) \rho_{3}(z, t)+\int_{\mathbb{R}^{2 d}} K_{1}(x, y) m_{1}(z \mid x, y) \rho_{1}(x, t) \rho_{2}(y, t) d x d y .
\end{aligned}
$$

Recall that the detailed balance condition gives

$$
\hat{K}_{1}(x-y) m_{1}(z \mid x, y) \times K_{\mathrm{d}}=K_{2}(z) m_{2}(x, y \mid z),
$$

where $K_{\mathrm{d}}=k_{2} / k_{1}$ is the equilibrium dissociation constant of the reaction and $K_{2}(z)=k_{2}$ is a constant function. Let us denote $(f * g)(x)=\int_{\mathbb{R}^{d}} f(x-y) g(y) d y$ as the convolution. Utilizing the detailed balance condition and the explicit form $m_{1}(z \mid x, y)=\alpha \delta(z-x)+(1-\alpha) \delta(z-y)$, Eq. (5.2) can be further simplified to

$$
\begin{aligned}
& \partial_{t} \rho_{1}=D^{\mathrm{A}} \Delta \rho_{1}-\left(\hat{K}_{1} * \rho_{2}\right) \rho_{1}+\alpha K_{2} \times \rho_{3}+(1-\alpha) K_{\mathrm{d}} \times\left(\hat{K}_{1} * \rho_{3}\right), \\
& \partial_{t} \rho_{2}=D^{\mathrm{B}} \Delta \rho_{2}-\left(\hat{K}_{1} * \rho_{1}\right) \rho_{2}+\alpha K_{\mathrm{d}} \times\left(\hat{K}_{1} * \rho_{3}\right)+(1-\alpha) K_{2} \times \rho_{3}, \\
& \partial_{t} \rho_{3}=D^{\mathrm{C}} \Delta \rho_{3}-K_{2} \rho_{3}+\alpha\left(\hat{K}_{1} * \rho_{2}\right) \rho_{1}+(1-\alpha)\left(\hat{K}_{1} * \rho_{1}\right) \rho_{2} .
\end{aligned}
$$

By summing up Eqs. (5.3) to (5.5), we see immediately

$$
\begin{aligned}
\partial_{t} \rho_{3}-D^{\mathrm{C}} \Delta \rho_{3}=- & \alpha\left(\partial_{t} \rho_{1}-D^{\mathrm{A}} \Delta \rho_{1}\right)-(1-\alpha)\left(\partial_{t} \rho_{2}-D^{\mathrm{B}} \Delta \rho_{2}\right) \\
& +\alpha^{2} K_{2} \times \rho_{3}+(1-\alpha)^{2} K_{2} \times \rho_{3}+2 \alpha(1-\alpha) K_{\mathrm{d}} \times\left(\hat{K}_{1} * \rho_{3}\right)-K_{2} \rho_{3} .
\end{aligned}
$$

Let $C$ and $C_{1}$ be a generic constant that only depends on $p, \alpha, K_{\mathrm{d}},\left\|\hat{K}_{1}\right\|_{L^{1}}, K_{2}, D^{\mathrm{A}}, D^{\mathrm{B}}, D^{\mathrm{C}}, \rho_{1}(x, 0), \rho_{2}(x, 0), \rho_{3}(x, 0)$. We'll always assume that for all $i=1,2,3,\left\|\rho_{i}(x, 0)\right\|_{L^{p}}<\infty$ for all $1 \leq p \leq \infty$ and $\rho_{i}(x, 0) \geq 0$, for all $x \in \mathbb{R}^{d}$. By the positivity preserving property of Eq. (5.2), we actually know that $\rho_{i}(x, t) \geq 0$, for all $t \in[0, T]$. Then Eq. (5.3) gives

$$
\partial_{t} \rho_{1}-D^{\mathrm{A}} \Delta \rho_{1} \leq \alpha K_{2} \times \rho_{3}+(1-\alpha) K_{\mathrm{d}} \times\left(\hat{K}_{1} * \rho_{3}\right),
$$

from which we obtain that for all $p \in(1, \infty)$,

$$
\left\|\rho_{1}(t)\right\|_{L^{p}} \leq\left\|\rho_{1}(0)\right\|_{L^{p}}+C \int_{0}^{t}\left[\left\|\rho_{3}(s)\right\|_{L^{p}}+\left\|\hat{K}_{1} * \rho_{3}(s)\right\|_{L^{p}}\right] d s .
$$

Using Young's inequality, $\left\|\hat{K}_{1} * \rho_{3}\right\|_{L^{p}} \leq\left\|\hat{K}_{1}\right\|_{L^{1}}\left\|\rho_{3}\right\|_{L^{p}}$, it further simplifies to

$$
\left\|\rho_{1}(t)\right\|_{L^{p}} \leq\left\|\rho_{1}(0)\right\|_{L^{p}}+C \int_{0}^{t}\left\|\rho_{3}(s)\right\|_{L^{p}} d s .
$$

Similarly, Eq. (5.4) gives

$$
\left\|\rho_{2}(t)\right\|_{L^{p}} \leq\left\|\rho_{2}(0)\right\|_{L^{p}}+C \int_{0}^{t}\left\|\rho_{3}(s)\right\|_{L^{p}} d s .
$$

Let $Q_{t}:=\mathbb{R}^{d} \times[0, t]$. Lemma 5.4 (an extension of Lemma 3.4 in $\left.[\mathrm{P} 10]\right)$ gives that

$$
\left\|\rho_{3}\right\|_{L^{p}\left(Q_{t}\right)} \leq C\left(1+\left\|\rho_{1}\right\|_{L^{p}\left(Q_{t}\right)}+\left\|\rho_{2}\right\|_{L^{p}\left(Q_{t}\right)}\right) .
$$

This implies

$$
\left(\int_{0}^{t}\left\|\rho_{3}(s)\right\|_{L^{p}} d s\right)^{p} \leq t^{p-1} \int_{Q_{t}}\left|\rho_{3}\right|^{p} \leq C\left(1+\left\|\rho_{1}\right\|_{L^{p}\left(Q_{t}\right)}+\left\|\rho_{2}\right\|_{L^{p}\left(Q_{t}\right)}\right)^{p}
$$




$$
\leq C\left(1+\left\|\rho_{1}\right\|_{L^{p}\left(Q_{t}\right)}^{p}+\left\|\rho_{2}\right\|_{L^{p}\left(Q_{t}\right)}^{p}\right) .
$$

Therefore, Eqs. (5.7) and (5.8) can be combined to give

$$
\begin{aligned}
\left\|\rho_{1}(t)\right\|_{L^{p}}^{p}+\left\|\rho_{2}(t)\right\|_{L^{p}}^{p} & \leq C_{1}+C\left(\int_{0}^{t}\left\|\rho_{3}(s)\right\|_{L^{p}} d s\right)^{p} \\
& \leq C_{1}+C\left(1+\int_{0}^{t}\left\|\rho_{1}(s)\right\|_{L^{p}}^{p}+\left\|\rho_{2}(s)\right\|_{L^{p}}^{p} d s\right) .
\end{aligned}
$$

By Gronwall's inequality, we know that $\rho_{1}, \rho_{2} \in L^{p}\left(Q_{T}\right)$, and furthermore $\rho_{3} \in L^{p}\left(Q_{T}\right)$ by Eq. (5.9), for all $p \in(1, \infty)$. Notice that since the reaction terms in Eqs. (5.3) to (5.5) are all polynomial (with a convolution that does not effect the $L^{p}$ bound by Young's inequality), they are bounded in $L^{p}\left(Q_{T}\right)$ for all $p \in(1, \infty)$. Morrey's inequality in $\mathbb{R}^{d+1}$ together with the $L^{p}$-regularity theory for parabolic operators (see Chapter IV Section 3 in [LSU68]) implies that $\rho_{1}, \rho_{2}, \rho_{3} \in L^{\infty}\left(Q_{T}\right)$, and therefore $T=\infty$ (See Lemma 1.1 in $[\mathrm{P} 10]$ ).

Lemma 5.4. For $\rho_{1}, \rho_{2}, \rho_{3}$ as used in Lemma 5.3 satisfying

$$
\partial_{t} \rho_{3}-D^{C} \Delta \rho_{3}+\theta_{1} \rho_{3}+\theta_{2}\left(\hat{K}_{1} * \rho_{3}\right)=-\alpha\left(\partial_{t} \rho_{1}-D^{A} \Delta \rho_{1}\right)-(1-\alpha)\left(\partial_{t} \rho_{2}-D^{B} \Delta \rho_{2}\right),
$$

on $Q_{T}:=\mathbb{R}^{d} \times[0, T]$ for some $\theta_{1} \in \mathbb{R}$ and $\theta_{2}<0$, it holds that

$$
\left\|\rho_{3}\right\|_{L^{p}\left(Q_{t}\right)} \leq C\left(1+\left\|\rho_{1}\right\|_{L^{p}\left(Q_{t}\right)}+\left\|\rho_{2}\right\|_{L^{p}\left(Q_{t}\right)}\right)
$$

for all $t \in(0, T]$ and $p \in(1, \infty)$.

Proof. For any $t \in(0, T]$, let $\phi$ be the solution of the following dual problem

$$
-\left[\partial_{t} \phi+D^{\mathrm{C}} \Delta \phi\right]+\theta_{1} \phi+\theta_{2}\left(\hat{K}_{1} * \phi\right)=\Theta
$$

with $\phi(t)=0$, where $\Theta \geq 0$ and $\Theta \in C_{0}^{\infty}\left(Q_{t}\right)$. Let $q=p /(p-1)$ be the conjugate index of $p$. Then the solution to Eq. (5.11) $\phi$ is smooth, satisfies $\phi \geq 0$ and for all $t \in[0, T]$,

$$
\left\|\phi_{t}\right\|_{L^{q}\left(Q_{t}\right)}+\|\Delta \phi\|_{L^{q}\left(Q_{t}\right)}+\sup _{s \in[0, t]}\|\phi(s)\|_{L^{q}}+\sup _{s \in[0, t]}\left\|\hat{K}_{1} * \phi(s)\right\|_{L^{q}} \leq C\|\Theta\|_{L^{q}\left(Q_{t}\right)} .
$$

One can show that $\phi \in L^{q}\left(Q_{t}\right)$ by a fixed point argument, $\phi \geq 0$ by comparison principle and further get the estimates Eq. (5.12) by investigating the mild solution form via Young's convolution inequality and $L^{q}$ estimates of heat potential (see Chapter IV Section 3 in [LSU68]).

Multiply $\partial_{t} \rho_{1}-D^{\mathrm{A}} \Delta \rho_{1}$ by $\phi$ and integrate by parts on $Q_{t}$, we find

$$
\begin{aligned}
\int_{Q_{t}}\left(\partial_{t} \rho_{1}-D^{\mathrm{A}} \Delta \rho_{1}\right) \phi d t d x & =\int_{\mathbb{R}^{d}}\left[\rho_{1}(t) \phi(t)-\rho_{1}(0) \phi(0)\right] d x-\int_{Q_{t}}\left(\partial_{t} \phi+D^{\mathrm{A}} \Delta \phi\right) \rho_{1} d t d x \\
& =-\int_{\mathbb{R}^{d}} \rho_{1}(0) \phi(0) d x+\int_{Q_{t}}\left(\Theta+\left(D^{\mathrm{C}}-D^{\mathrm{A}}\right) \Delta \phi-\theta_{1} \phi-\theta_{2}\left(\hat{K}_{1} * \phi\right)\right) \rho_{1} d t d x .
\end{aligned}
$$

Multipling Eq. (5.10) by $\phi$ and integrating by parts over $Q_{t}$ in a similar manner as Eq. (5.13), we find

$$
\begin{aligned}
\int_{Q_{t}} \Theta \times \rho_{3} d t d x=\int_{\mathbb{R}^{d}} \rho_{3}(0) \phi(0) d x+\alpha \int_{\mathbb{R}^{d}} \rho_{1}(0) \phi(0) d x+(1-\alpha) \int_{\mathbb{R}^{d}} \rho_{2}(0) \phi(0) d x \\
-\alpha \int_{Q_{t}}\left(\Theta+\left(D^{\mathrm{C}}-D^{\mathrm{A}}\right) \Delta \phi-\theta_{1} \phi-\theta_{2}\left(\hat{K}_{1} * \phi\right)\right) \rho_{1} d t d x \\
-(1-\alpha) \int_{Q_{t}}\left(\Theta+\left(D^{\mathrm{C}}-D^{\mathrm{B}}\right) \Delta \phi-\theta_{1} \phi-\theta_{2}\left(\hat{K}_{1} * \phi\right)\right) \rho_{2} d t d x .
\end{aligned}
$$

Notice that $\rho_{1}, \rho_{2}, \rho_{3}, \phi \geq 0$, then Eq. (5.14) combined with Eq. (5.12) gives

$$
\int_{Q_{t}} \Theta \times \rho_{3} \leq C|| \Theta \|_{L^{q}\left(Q_{t}\right)}\left(1+\left\|\rho_{1}\right\|_{L^{p}\left(Q_{t}\right)}+\left\|\rho_{2}\right\|_{L^{p}\left(Q_{t}\right)}\right) d t d x .
$$

Since the choices of $\Theta$ are arbitrary, by duality argument, we have

$$
\left\|\rho_{3}\right\|_{L^{p}\left(Q_{t}\right)} \leq C\left(1+\left\|\rho_{1}\right\|_{L^{p}\left(Q_{t}\right)}+\left\|\rho_{2}\right\|_{L^{p}\left(Q_{t}\right)}\right) .
$$




\section{A Lemmas for Estimating Differences Between Two Models}

First, we start with a key estimate presented in Lemma A.1.

Lemma A.1. For any non-negative kernel $\hat{K}^{\epsilon}(w) \geq 0$ with $w \in \mathbb{R}^{d}, \epsilon$ sufficiently small, of the type

(A) : radially symmetric $\hat{K}^{\epsilon}(w)$ only depends on $|w|$,

(B) : renormalized integrable $\left\|\hat{K}^{\epsilon}\right\|_{L^{1}\left(\mathbb{R}^{d}\right)}=1$,

(C): $\int_{\mathbb{R}^{d}} \hat{K}^{\epsilon}(w)|w|^{2} d w=O\left(\epsilon^{2}\right)$,

and for any function $f, g \in C_{b}^{2}\left(\mathbb{R}^{d}\right)$, any real number $|\alpha|<\infty$, we get as $\epsilon \rightarrow 0$

$$
\left\|\int_{\mathbb{R}^{d}} \hat{K}^{\epsilon}(x-y) f(x+\alpha(y-x)) g(y) d y-f(x) g(x)\right\|_{L^{\infty}}=\|f \times g\|_{C_{b}^{2}\left(\mathbb{R}^{d}\right)} O\left(\epsilon^{2}\right),
$$

where $O\left(\epsilon^{2}\right)$ depends on $\alpha$.

Remark A.2. In particular, in Lemma A.1, one can choose $g \equiv 1$ to obtain as $\epsilon \rightarrow 0$

$$
\left\|\int_{\mathbb{R}^{d}} \hat{K}^{\epsilon}(x-y) f(x+\alpha(y-x)) d y-f(x)\right\|_{L^{\infty}}=\|f\|_{C_{b}^{2}\left(\mathbb{R}^{d}\right)} O\left(\epsilon^{2}\right) .
$$

Proof of Lemma A.1. Assume that $\alpha \neq 0$. The case when $\alpha=0$ can be adapted from the following proof. Notice that for any $f \in C_{b}^{2}\left(\mathbb{R}^{d}\right)$, any $x, y \in \mathbb{R}^{d}$, we can apply Taylor's theorem to obtain

$$
f(y)=f(x)+\langle\nabla f(x), y-x\rangle+\left\langle y-x, D^{2} f(\xi)(y-x)\right\rangle,
$$

where $\langle\cdot, \cdot\rangle$ is the inner product on $\mathbb{R}^{d}, D^{2} f(\xi)$ is the Hessen matrix at $x$ and $\xi \in B_{|y-x|}(x)$. Since $f \in C_{b}^{2}\left(\mathbb{R}^{d}\right)$, we can alternatively rewrite Eq. (A.3) as

$$
|f(y)-f(x)-\langle\nabla f(x), y-x\rangle| \leq\|f\|_{C_{b}^{2}\left(\mathbb{R}^{d}\right)} \times|y-x|^{2} .
$$

Applying Taylor's theorem to $f(x+\alpha(y-x)) \times g(y)$, we find

$$
\begin{aligned}
& |f(x+\alpha(y-x)) g(y)-f(x) g(x)-\alpha\langle\nabla f(x),(y-x)\rangle g(x)-\langle\nabla g(x),(y-x)\rangle f(x)| \\
& \leq\left(\alpha^{2} \vee 1\right)\|f \times g\|_{C_{b}^{2}\left(\mathbb{R}^{d}\right)} \times|y-x|^{2} .
\end{aligned}
$$

so that

$$
\begin{aligned}
& \left\|\int_{\mathbb{R}^{d}} \hat{K}^{\epsilon}(x-y) f(x+\alpha(y-x)) g(y) d y-f(x) g(x)\right\|_{L^{\infty}} \\
& =\sup _{x \in \mathbb{R}^{d}}\left|\int_{\mathbb{R}^{d}} \hat{K}^{\epsilon}(x-y)[f(x+\alpha(y-x)) g(y)-f(x) g(x)] d y\right| \\
& \leq \sup _{x \in \mathbb{R}^{d}}\left|\int_{\mathbb{R}^{d}} \hat{K}^{\epsilon}(x-y)[f(x+\alpha(y-x)) g(y)-f(x) g(x)-\alpha\langle\nabla f(x),(y-x)\rangle g(x)-\langle\nabla g(x),(y-x)\rangle f(x)] d y\right| \\
& \quad+\sup _{x \in \mathbb{R}^{d}}\left|\int_{\mathbb{R}^{d}} \hat{K}^{\epsilon}(x-y)(\alpha\langle\nabla f(x),(y-x)\rangle g(x)+\langle\nabla g(x),(y-x)\rangle f(x)) d y\right| \\
& \leq \sup _{x \in \mathbb{R}^{d}} \int_{\mathbb{R}^{d}} \hat{K}^{\epsilon}(x-y)\left(\alpha^{2} \vee 1\right)\|f \times g\|_{C_{b}^{2}\left(\mathbb{R}^{d}\right)} \times|y-x|^{2} d y \quad \text { (by property (A) of the reaction kernel ) } \\
& \leq\left(\alpha^{2} \vee 1\right)\|f \times g\|_{C_{b}^{2}\left(\mathbb{R}^{d}\right)} \int_{\mathbb{R}^{d}} \hat{K}^{\epsilon}(w)|w|^{2} d w, \quad(\text { by property (C) of the reaction kernel ) } \\
& \leq\|f \times g\|_{C_{b}^{2}\left(\mathbb{R}^{d}\right)} O\left(\epsilon^{2}\right),
\end{aligned}
$$

where we note that in the final expression the $O\left(\epsilon^{2}\right)$ term depends on $\alpha$.

\section{Lemma A.3.}

$$
\Lambda^{\ell, j}(\tau) \leq 2 C\left(\max _{j=1, \cdots, J}\left\|\rho_{j}(x, \tau)-\rho_{j}^{\epsilon}(x, \tau)\right\|_{L^{\infty}}+\left(C_{2}+\frac{C_{3}}{\sqrt{\tau}}\right) O\left(\epsilon^{2}\right)\right),
$$

where $C, C_{2}, C_{3}$ is defined in the proof of Theorem 3.1 and independent of $\epsilon$. 
Proof. We'll discuss this case by case. When $\alpha_{\ell j}=0, \Lambda^{\ell, j}(\tau)=0$, and Eq. (A.5) is trivially true. When $\alpha_{\ell j}=1$, note that $\boldsymbol{\alpha}^{(\ell)} !=1$ for the allowable types of reactions. If $\mathcal{R}_{\ell}$ is a first order reaction, based on Assumption 2.5, $K_{\ell}^{\epsilon}(x)=k_{\ell}, x \in \mathbb{R}^{d}$, is a constant rate function. As long as we choose $k_{\ell}=\kappa_{\ell}$,

$$
\Lambda^{\ell, j}(\tau) \leq k_{\ell} \max _{j=1, \cdots, J}\left\|\rho_{j}(x, \tau)-\rho_{j}^{\epsilon}(x, \tau)\right\|_{L^{\infty}}
$$

When $\alpha_{\ell j}=1$ and $\mathcal{R}_{\ell}$ is of the type $S_{i}+S_{j} \rightarrow \cdots, i \neq j$, Assumption 2.3 gives

$$
\begin{aligned}
\Lambda^{\ell, j}(\tau)= & \left\|\left(\int_{\mathbb{R}^{d}} \hat{K}_{\ell}^{\epsilon}(x-y) \rho_{i}^{\epsilon}(y, \tau) d y\right) \rho_{j}^{\epsilon}(x, \tau)-\kappa_{\ell} \rho_{i}(x, \tau) \rho_{j}(x, \tau)\right\|_{L^{\infty}} \\
\leq \| & \left(\int_{\mathbb{R}^{d}} \hat{K}_{\ell}^{\epsilon}(x-y) \rho_{i}^{\epsilon}(y, \tau) d y-\kappa_{\ell} \rho_{i}^{\epsilon}(x, \tau)\right) \rho_{j}^{\epsilon}(x, \tau) \|_{L^{\infty}} \\
& \quad\|\| \rho_{j}^{\epsilon}(x, \tau)\left(\rho_{i}^{\epsilon}(x, \tau)-\rho_{i}(x, \tau)\right)\left\|_{L^{\infty}}+\right\| \rho_{i}(x, \tau)\left(\rho_{j}^{\epsilon}(x, \tau)-\rho_{j}(x, \tau)\right) \|_{L^{\infty}} \\
\leq \| & \rho_{i}^{\epsilon}(x, \tau)\left\|_{C_{b}^{2}\left(\mathbb{R}^{d}\right)} O\left(\epsilon^{2}\right) \times\right\| \rho_{j}^{\epsilon}(x, \tau)\left\|_{L^{\infty}}+\right\| \rho_{j}^{\epsilon}(x, \tau)\left\|_{L^{\infty}}\right\| \rho_{i}^{\epsilon}(x, \tau)-\rho_{i}(x, \tau) \|_{L^{\infty}} \\
& +\left\|\rho_{i}(x, \tau)\right\|_{L^{\infty}}\left\|\rho_{j}^{\epsilon}(x, \tau)-\rho_{j}(x, \tau)\right\|_{L^{\infty}} \\
\leq & C\left(\left\|\rho_{i}^{\epsilon}(x, \tau)\right\|_{C_{b}^{2}\left(\mathbb{R}^{d}\right)} O\left(\epsilon^{2}\right)+2 \max _{j=1, \cdots, J}\left\|\rho_{j}^{\epsilon}(x, \tau)-\rho_{j}(x, \tau)\right\|_{L^{\infty}}\right),
\end{aligned}
$$

by choosing $k_{\ell}=\kappa_{\ell}$ and applying Lemma A.1 and the regularity results of the solutions in Theorem 4.6 and Theorem 4.4. Here in the last step, recall that $C=\left(\max _{\ell=1, \cdots, L} k_{\ell}\right) \max _{j=1, \cdots, J}\left\{\sup _{\epsilon>0} \sup _{\tau \in\left[0, T_{0}\right]}\left\|\rho_{j}^{\epsilon}(x, \tau)\right\|_{L^{\infty}} \mathrm{V}\right.$ $\left.\sup _{\tau \in\left[0, T_{0}\right]}\left\|\rho_{j}(x, \tau)\right\|_{L^{\infty}}\right\}$ was assumed independent of $\epsilon$.

When $\alpha_{\ell j}=2, \mathcal{R}_{\ell}$ should be of the type $S_{j}+S_{j} \rightarrow \cdots$, Assumption 2.3 gives

$$
\Lambda^{\ell, j}(\tau)=\left\|\left(\int_{\mathbb{R}^{d}} \hat{K}_{\ell}^{\epsilon}(x-y) \rho_{j}^{\epsilon}(y, \tau) d y\right) \rho_{j}^{\epsilon}(x, \tau)-2 \kappa_{\ell} \rho_{j}(x, \tau)^{2}\right\|_{L^{\infty}} .
$$

By choosing $k_{\ell}=2 \kappa_{\ell}$ in this case, Eq. (A.5) follows closely from Eq. (A.6).

\section{Lemma A.4.}

$$
\Theta^{\ell, j}(\tau) \leq 4 C\left(\max _{j=1, \cdots, J}\left\|\rho_{j}(x, \tau)-\rho_{j}^{\epsilon}(x, \tau)\right\|_{L^{\infty}}+\left(C_{2}+\frac{C_{3}}{\sqrt{\tau}}\right) O\left(\epsilon^{2}\right)\right),
$$

where $C, C_{2}, C_{3}$ is defined in the proof of Theorem 3.1 and independent of $\epsilon$.

Proof. We'll again discuss this case by case. When $\beta_{\ell j}=0$ then $\Theta^{\ell, j}(\tau)=0$, and Eq. (A.7) is trivially true. (1) When $\mathcal{R}_{\ell}$ is of the type $S_{i} \rightarrow S_{j}, i \neq j$, by plugging in Assumption 2.7 and Assumption 2.5, we obtain

$$
\Theta^{\ell, j}(\tau)=\left\|k_{\ell} \rho_{i}^{\epsilon}(x, \tau)-\kappa_{\ell} \rho_{i}(x, \tau)\right\|_{L^{\infty}} \leq k_{\ell}\left\|\rho_{i}^{\epsilon}(x, \tau)-\rho_{i}(x, \tau)\right\|_{L^{\infty}} .
$$

(2) When $\mathcal{R}_{\ell}$ is of the type $S_{i}+S_{k} \rightarrow S_{j}, i \neq k$, by plugging in Assumption 2.8 and Assumption 2.3, we obtain

$$
\begin{aligned}
\Theta^{\ell, j}(\tau)= & \left\|\int_{\mathbb{R}^{2 d}} \hat{K}_{\ell}^{\epsilon}\left(x_{1}-x_{2}\right) m_{\ell}^{\epsilon}\left(x \mid x_{1}, x_{2}\right) \rho_{i}^{\epsilon}\left(x_{1}, \tau\right) \rho_{k}^{\epsilon}\left(x_{2}, \tau\right) d x_{1} d x_{2}-\kappa_{\ell} \rho_{i}(x, \tau) \rho_{k}(x, \tau)\right\|_{L^{\infty}} \\
\leq \| & \left\|\int_{\mathbb{R}^{2 d}} \hat{K}_{\ell}^{\epsilon}\left(x_{1}-x_{2}\right) m_{\ell}^{\epsilon}\left(x \mid x_{1}, x_{2}\right) \rho_{i}^{\epsilon}\left(x_{1}, \tau\right) \rho_{k}^{\epsilon}\left(x_{2}, \tau\right) d x_{1} d x_{2}-\kappa_{\ell} \rho_{i}^{\epsilon}(x, \tau) \rho_{k}^{\epsilon}(x, \tau)\right\|_{L^{\infty}} \\
& \quad+\kappa_{\ell}\left\|\rho_{i}^{\epsilon}(x, \tau)\right\|_{L^{\infty}}\left\|\rho_{k}(x, \tau)-\rho_{k}^{\epsilon}(x, \tau)\right\|_{L^{\infty}}+\kappa_{\ell}\left\|\rho_{i}^{\epsilon}(x, \tau)-\rho_{i}(x, \tau)\right\|_{L^{\infty}}\left\|\rho_{k}(x, \tau)\right\|_{L^{\infty}} .
\end{aligned}
$$

Without loss of generality, we assume that $\alpha_{i}>0$ for all $1 \leq i \leq I$ in the following, then by choosing $k_{\ell}=\kappa_{\ell}$, we have that

$$
\begin{aligned}
& \left|\int_{\mathbb{R}^{2 d}} \hat{K}_{\ell}^{\epsilon}\left(x_{1}-x_{2}\right) m_{\ell}^{\epsilon}\left(x \mid x_{1}, x_{2}\right) \rho_{i}^{\epsilon}\left(x_{1}, \tau\right) \rho_{k}^{\epsilon}\left(x_{2}, \tau\right) d x_{1} d x_{2}-\kappa_{\ell} \rho_{i}^{\epsilon}(x, \tau) \rho_{k}^{\epsilon}(x, \tau)\right| \\
& \quad=\left|\int_{\mathbb{R}^{2 d}} \hat{K}_{\ell}^{\epsilon}\left(x_{1}-x_{2}\right) \sum_{i=1}^{I} p_{i} \delta\left(x-\left(\alpha_{i} x_{1}+\left(1-\alpha_{i}\right) x_{2}\right)\right) \rho_{i}^{\epsilon}\left(x_{1}, \tau\right) \rho_{k}^{\epsilon}\left(x_{2}, \tau\right) d x_{1} d x_{2}-\kappa_{\ell} \rho_{i}^{\epsilon}(x, \tau) \rho_{k}^{\epsilon}(x, \tau)\right| \\
& \quad \leq \sum_{i=1}^{I} p_{i}\left|\int_{\mathbb{R}^{d}} \frac{1}{\alpha_{i}^{d}} \hat{K}_{\ell}^{\epsilon}\left(\frac{1}{\alpha_{i}}\left(x-x_{2}\right)\right) \rho_{i}^{\epsilon}\left(\frac{1}{\alpha_{i}}\left(x-\left(1-\alpha_{i}\right) x_{2}\right), \tau\right) \rho_{k}^{\epsilon}\left(x_{2}, \tau\right) d x_{2}-k_{1} \rho_{i}^{\epsilon}(x, \tau) \rho_{k}^{\epsilon}(x, \tau)\right|
\end{aligned}
$$




$$
\begin{aligned}
& =\sum_{i=1}^{I} p_{i}\left|\int_{\mathbb{R}^{d}} \frac{1}{\alpha_{i}^{d}} \hat{K}_{\ell}^{\epsilon}\left(\frac{1}{\alpha_{i}}\left(x-x_{2}\right)\right) \rho_{i}^{\epsilon}\left(x+\frac{\left(1-\alpha_{i}\right)}{\alpha_{i}}\left(x-x_{2}\right), \tau\right) \rho_{k}^{\epsilon}\left(x_{2}, \tau\right) d x_{2}-\kappa_{\ell} \rho_{i}^{\epsilon}(x, \tau) \rho_{k}^{\epsilon}(x, \tau)\right| \\
& =\left\|\rho_{i}^{\epsilon}(x, \tau) \rho_{k}^{\epsilon}(x, \tau)\right\|_{C_{b}^{2}\left(\mathbb{R}^{d}\right)} O\left(\epsilon^{2}\right),
\end{aligned}
$$

based on Lemma A.1 and the regularity results in Theorem 4.6 and Theorem 4.4. Substituting this back into Eq. (A.9), we obtain

$$
\begin{array}{rl}
\Theta^{\ell, j}(\tau) \leq & k_{\ell}\left\|\rho_{i}^{\epsilon}(x, \tau)\right\|\left\|_{L^{\infty}}\right\| \rho_{k}(x, \tau)-\rho_{k}^{\epsilon}(x, \tau)\left\|_{L^{\infty}}+k_{\ell}\right\| \rho_{i}^{\epsilon}(x, \tau)-\rho_{i}(x, \tau)\left\|_{L^{\infty}}\right\| \rho_{k}(x, \tau) \|_{L^{\infty}} \\
& +\left\|\rho_{i}^{\epsilon}(x, \tau) \rho_{k}^{\epsilon}(x, \tau)\right\|_{C_{b}^{2}\left(\mathbb{R}^{d}\right)} O\left(\epsilon^{2}\right) \\
\leq 2 & 2 C\left(\max _{j=1, \cdots, J}\left\|\rho_{j}(x, \tau)-\rho_{j}^{\epsilon}(x, \tau)\right\|_{L^{\infty}}+\left\|\rho_{i}^{\epsilon}(x, \tau) \rho_{k}^{\epsilon}(x, \tau)\right\|_{C_{b}^{2}\left(\mathbb{R}^{d}\right)} O\left(\epsilon^{2}\right)\right)
\end{array}
$$

where $C=\left(\max _{\ell=1, \cdots, L} k_{\ell}\right) \max _{j=1, \cdots, J}\left\{\sup _{\epsilon>0} \sup _{\tau \in\left[0, T_{0}\right]}\left\|\rho_{j}^{\epsilon}(x, \tau)\right\|_{L^{\infty}} \vee \sup _{\tau \in\left[0, T_{0}\right]}\left\|\rho_{j}(x, \tau)\right\|_{L^{\infty}}\right\}$ is independent of $\epsilon$.

(3) When $\mathcal{R}_{\ell}$ is of the type $S_{i}+S_{i} \rightarrow S_{j}$, by plugging in Assumption 2.8 and Assumption 2.3, we obtain

$$
\Theta^{\ell, j}(\tau)=\left\|\frac{1}{2} \int_{\mathbb{R}^{2 d}} \hat{K}_{\ell}^{\epsilon}\left(x_{1}-x_{2}\right) m_{\ell}^{\epsilon}\left(x \mid x_{1}, x_{2}\right) \rho_{i}^{\epsilon}\left(x_{1}, \tau\right) \rho_{i}^{\epsilon}\left(x_{2}, \tau\right) d x_{1} d x_{2}-\kappa_{\ell} \rho_{i}(x, \tau) \rho_{i}(x, \tau)\right\|_{L^{\infty}} .
$$

By choosing $k_{\ell}=2 \kappa_{\ell}$ and following the same arguments as the previous case, we shall obtain

$$
\Theta^{\ell, j}(\tau) \leq 2 C\left(\max _{j=1, \cdots, J}\left\|\rho_{j}(x, \tau)-\rho_{j}^{\epsilon}(x, \tau)\right\|_{L^{\infty}}+\left\|\rho_{i}^{\epsilon}(x, \tau) \rho_{k}^{\epsilon}(x, \tau)\right\|_{C_{b}^{2}\left(\mathbb{R}^{d}\right)} O\left(\epsilon^{2}\right)\right) .
$$

(4) When $\mathcal{R}_{\ell}$ is of the type $S_{i} \rightarrow S_{j}+S_{k}$, by plugging in Assumption 2.10 and Assumption 2.5, using symmetry and choosing $k_{\ell}=\kappa_{\ell}$ we obtain

$$
\begin{aligned}
\Theta^{\ell, j}(\tau)= & \beta_{\ell j}\left\|\left(\int_{\mathbb{R}^{d}} \hat{K}_{\ell}^{\epsilon}(z)\left(\int_{\mathbb{R}^{d}} m_{\ell}^{\epsilon}(x, y \mid z) d y\right) \rho_{i}^{\epsilon}(z, \tau) d z\right)-\kappa_{\ell} \rho_{i}(x, \tau)\right\|_{L^{\infty}} \\
= & \beta_{\ell j} \kappa_{\ell}\left\|\left(\int_{\mathbb{R}^{d}}\left(\int_{\mathbb{R}^{d}} \rho^{\epsilon}(|x-y|) \sum_{j=1}^{J} q_{j} \times \delta\left(z-\left(\beta_{j} x+\left(1-\beta_{j}\right) y\right)\right) d y\right) \rho_{i}^{\epsilon}(z, \tau) d z\right)-\rho_{i}(x, \tau)\right\|_{L^{\infty}} \\
= & \beta_{\ell j} \kappa_{\ell}\left\|\sum_{j=1}^{J} q_{j} \times \int_{\mathbb{R}^{d}} \rho^{\epsilon}(|x-y|) \rho_{i}^{\epsilon}\left(\beta_{j} x+\left(1-\beta_{j}\right) y, \tau\right) d y-\rho_{i}(x, \tau)\right\|_{L^{\infty}} \\
\leq & \beta_{\ell j} \kappa_{\ell}\left\|\sum_{j=1}^{J} q_{j} \times \int_{\mathbb{R}^{d}} \rho^{\epsilon}(|x-y|)\left(\rho_{i}^{\epsilon}\left(\beta_{j} x+\left(1-\beta_{j}\right) y, \tau\right)-\rho_{i}\left(\beta_{j} x+\left(1-\beta_{j}\right) y, \tau\right)\right) d y\right\|_{L^{\infty}} \\
& \quad+\beta_{\ell j} \kappa_{\ell}\left\|\sum_{j=1}^{J} q_{j} \times \int_{\mathbb{R}^{d}} \rho^{\epsilon}(|x-y|) \rho_{i}\left(\beta_{j} x+\left(1-\beta_{j}\right) y, \tau\right) d y-\rho_{i}(x, \tau)\right\|_{L^{\infty}} \\
\leq & \beta_{\ell j} \kappa_{\ell}\left(\left\|\rho_{i}^{\epsilon}(x, \tau)-\rho_{i}(x, \tau)\right\|_{L^{\infty}}+\left\|\rho_{i}(x, \tau)\right\|_{C_{b}^{2}\left(\mathbb{R}^{d}\right)} O\left(\epsilon^{2}\right)\right)
\end{aligned}
$$

where the last term here again uses Lemma A.1 and the regularity of solutions in Theorem 4.6 and Theorem 4.4 .

(5) When $\mathcal{R}_{\ell}$ is of the type $S_{i}+S_{k} \rightarrow S_{j}+S_{r}, i \neq k$, by plugging in Assumption 2.9 and Assumption 2.3, using symmetry and choosing $k_{\ell}=\kappa_{\ell}$ we obtain

$$
\begin{aligned}
\Theta^{\ell, j}(\tau)= & \beta_{\ell j}\left\|\left(\int_{\mathbb{R}^{2 d}} K_{\ell}^{\epsilon}(z, w)\left(\int_{\mathbb{R}^{d}} m_{\ell}^{\epsilon}(x, y \mid z, w) d y\right) \rho_{i}^{\epsilon}(z, \tau) \rho_{k}^{\epsilon}(w, \tau) d z d w\right)-\kappa_{\ell} \rho_{i}(x, \tau) \rho_{k}(x, \tau)\right\|_{L^{\infty}} \\
= & \beta_{\ell j} p\left\|\left(\int_{\mathbb{R}^{d}} K_{\ell}^{\epsilon}(x, y) \rho_{i}^{\epsilon}(x, \tau) \rho_{k}^{\epsilon}(y, \tau) d y\right)-\kappa_{\ell} \rho_{i}(x, \tau) \rho_{k}(x, \tau)\right\|_{L^{\infty}} \\
& \quad+\beta_{\ell j}(1-p)\left\|\left(\int_{\mathbb{R}^{d}} K_{\ell}^{\epsilon}(y, x) \rho_{i}^{\epsilon}(y, \tau) \rho_{k}^{\epsilon}(x, \tau) d y\right)-\kappa_{\ell} \rho_{i}(x, \tau) \rho_{k}(x, \tau)\right\|_{L^{\infty}} \\
\leq & \beta_{\ell j} 2 C\left(\sup _{i=1, \cdots, J}\left\|\rho_{i}^{\epsilon}(x, \tau)\right\|_{C_{b}^{2}\left(\mathbb{R}^{d}\right)} O\left(\epsilon^{2}\right)+\max _{j=1, \cdots, J}\left\|\rho_{j}^{\epsilon}(x, \tau)-\rho_{j}(x, \tau)\right\|_{L^{\infty}}\right),
\end{aligned}
$$


where the last inequality comes from Eq. (A.6).

(6) When $\mathcal{R}_{\ell}$ is of the type $S_{i}+S_{i} \rightarrow S_{j}+S_{r}$, by choosing $k_{\ell}=2 \kappa_{\ell}$ instead, we obtain the same estimates as Eq. (A.12).

This concludes the proof of the lemma.

\section{B Lemmas for Estimating Derivatives of the Nonlinear Term of MFM}

Lemma B.1. For $\mathcal{R}_{\ell}$ to be a first or second order reactions,

$$
\left|\partial_{x_{i}} \int_{\tilde{\boldsymbol{x}} \in \mathbb{X}^{(\ell)}} \delta_{x}\left(\tilde{x}_{1}^{(j)}\right) K_{\ell}(\tilde{\boldsymbol{x}})\left(\Pi_{k=1}^{J} \Pi_{s=1}^{\alpha_{\ell k}} \rho_{k}\left(\tilde{x}_{s}^{(k)}, t\right)\right) d \tilde{\boldsymbol{x}}\right| \leq k_{\ell}\left(2 C_{2} \vee 1\right) \sup _{k=1, \cdots, J}\left\|\partial_{x_{i}} \rho_{k}(x, t)\right\|_{C_{b}\left(\mathbb{R}^{d}\right)} .
$$

Proof. When $\mathcal{R}_{\ell}$ is first order of the type $S_{j} \rightarrow \cdots$,

$$
\begin{aligned}
\left|\partial_{x_{i}} \int_{\tilde{\boldsymbol{x}} \in \mathbb{X}(\ell)} \delta_{x}\left(\tilde{x}_{1}^{(j)}\right) K_{\ell}(\tilde{\boldsymbol{x}})\left(\Pi_{k=1}^{J} \Pi_{s=1}^{\alpha_{\ell k}} \rho_{k}\left(\tilde{x}_{s}^{(k)}, t\right)\right) d \tilde{\boldsymbol{x}}\right| & =k_{\ell}\left|\partial_{x_{i}} \rho_{j}(x, t)\right|, \\
& \leq k_{\ell} \sup _{k=1, \cdots, J}\left\|\partial_{x_{i}} \rho_{k}(x, t)\right\|_{C_{b}\left(\mathbb{R}^{d}\right)} .
\end{aligned}
$$

When $\mathcal{R}_{\ell}$ is second order of the type $S_{k}+S_{j} \rightarrow \cdots$,

$$
\begin{aligned}
& \left|\partial_{x_{i}} \int_{\tilde{\boldsymbol{x}} \in \mathbb{X}^{(\ell)}} \delta_{x}\left(\tilde{x}_{1}^{(j)}\right) K_{\ell}(\tilde{\boldsymbol{x}})\left(\Pi_{k=1}^{J} \Pi_{s=1}^{\alpha_{\ell k}} \rho_{k}\left(\tilde{x}_{s}^{(k)}, t\right)\right) d \tilde{\boldsymbol{x}}\right| \\
& =\left|\partial_{x_{i}}\left(\int_{\mathbb{R}^{d}} \hat{K}_{\ell}(x-y) \rho_{k}(y, t) d y \times \rho_{j}(x, t)\right)\right|, \\
& =\left|\left(\int_{\mathbb{R}^{d}} \hat{K}_{\ell}(y) \partial_{x_{i}} \rho_{k}(x-y, t) d y \times \rho_{j}(x, t)\right)\right|+\left|\left(\int_{\mathbb{R}^{d}} \hat{K}_{\ell}(y) \rho_{k}(x-y, t) d y \times \partial_{x_{i}} \rho_{j}(x, t)\right)\right|, \\
& \leq 2 k_{\ell} C_{2} \sup _{k=1, \cdots, J}\left\|\partial_{x_{i}} \rho_{k}(x, t)\right\|_{C_{b}\left(\mathbb{R}^{d}\right) .}
\end{aligned}
$$

Lemma B.2. For $\mathcal{R}_{\ell}$ to be a reaction producing one or two species,

$$
\begin{aligned}
& \left|\partial_{x_{m}} \int_{\tilde{\boldsymbol{x}} \in \mathbb{X}^{(\ell)}} K_{\ell}(\tilde{\boldsymbol{x}})\left(\int_{\boldsymbol{y} \in \mathbb{Y}^{(\ell)}} \delta_{x}\left(y_{1}^{(j)}\right) m_{\ell}(\boldsymbol{y} \mid \tilde{\boldsymbol{x}}) d \boldsymbol{y}\right)\left(\Pi_{k=1}^{J} \Pi_{s=1}^{\alpha_{\ell k}} \rho_{k}\left(\tilde{x}_{s}^{(k)}, t\right)\right) d \tilde{\boldsymbol{x}}\right| \\
& \leq k_{\ell}\left(2 C_{2} \vee 1\right) \sup _{k=1, \cdots, J}\left\|\partial_{x_{m}} \rho_{k}(x, t)\right\|_{C_{b}\left(\mathbb{R}^{d}\right)} .
\end{aligned}
$$

Proof. We discuss this case by case for the different types of reactions.

(1) When $\mathcal{R}_{\ell}$ is of the type $S_{k} \rightarrow S_{j}$,

$$
\begin{aligned}
& \left|\partial_{x_{m}} \int_{\tilde{\boldsymbol{x}} \in \mathbb{X}^{(\ell)}} K_{\ell}(\tilde{\boldsymbol{x}})\left(\int_{\boldsymbol{y} \in \mathbb{Y}^{(\ell)}} \delta_{x}\left(y_{1}^{(j)}\right) m_{\ell}(\boldsymbol{y} \mid \tilde{\boldsymbol{x}}) d \boldsymbol{y}\right)\left(\Pi_{k=1}^{J} \Pi_{s=1}^{\alpha_{\ell k}} \rho_{k}\left(\tilde{x}_{s}^{(k)}, t\right)\right) d \tilde{\boldsymbol{x}}\right| \\
& =k_{\ell}\left|\partial_{x_{m}} \rho_{k}(x, t)\right| \leq k_{\ell} \sup _{k=1, \cdots, J}\left\|\partial_{x_{m}} \rho_{k}(x, t)\right\|_{C_{b}\left(\mathbb{R}^{d}\right) .}
\end{aligned}
$$

(2) When $\mathcal{R}_{\ell}$ is of the type $S_{k}+S_{r} \rightarrow S_{j}$, without loss of generality, let us assume $\alpha_{i}>0$ for all $i=1, \cdots, I$. Then,

$$
\begin{aligned}
& \left|\partial_{x_{m}} \int_{\tilde{\boldsymbol{x}} \in \mathbb{X}(\ell)} K_{\ell}(\tilde{\boldsymbol{x}})\left(\int_{\boldsymbol{y} \in \mathbb{Y}(\ell)} \delta_{x}\left(y_{1}^{(j)}\right) m_{\ell}(\boldsymbol{y} \mid \tilde{\boldsymbol{x}}) d \boldsymbol{y}\right)\left(\Pi_{k=1}^{J} \Pi_{s=1}^{\alpha_{\ell k}} \rho_{k}\left(\tilde{x}_{s}^{(k)}, t\right)\right) d \tilde{\boldsymbol{x}}\right| \\
& =\left|\partial_{x_{m}} \int_{\mathbb{R}^{2 d}} \hat{K}_{\ell}(y-z) m_{\ell}(x \mid y, z) \rho_{k}(y, t) \rho_{r}(z, t) d y d z\right| . \\
& =\left|\partial_{x_{m}} \int_{\mathbb{R}^{2 d}} \hat{K}_{\ell}(y-z) \sum_{i=1}^{I} p_{i} \times \delta\left(x-\left(\alpha_{i} y+\left(1-\alpha_{i}\right) z\right)\right) \rho_{k}(y, t) \rho_{r}(z, t) d y d z\right| . \\
& =\left|\sum_{i=1}^{I} p_{i} \times \partial_{x_{m}} \int_{\mathbb{R}^{d}} \frac{1}{\alpha_{i}^{d}} \hat{K}_{\ell}\left(\frac{x-z}{\alpha_{i}}\right) \rho_{k}\left(\frac{x-\left(1-\alpha_{i}\right) z}{\alpha_{i}}, t\right) \rho_{r}(z, t) d z\right|,
\end{aligned}
$$




$$
\begin{aligned}
& =\left|\sum_{i=1}^{I} p_{i} \times \int_{\mathbb{R}^{d}} \hat{K}_{\ell}(w) \partial_{x_{m}}\left(\rho_{k}\left(x+\left(1-\alpha_{i}\right) w, t\right) \rho_{r}\left(x-\alpha_{i} w, t\right)\right) d w\right| \\
& \leq k_{\ell} \times 2 \sup _{k=1, \cdots, J}\left\|\partial_{x_{m}} \rho_{k}(x, t)\right\|_{C_{b}\left(\mathbb{R}^{d}\right)}\|\boldsymbol{\rho}\|\left\|_{M} \leq 2 k_{\ell} C_{2} \sup _{k=1, \cdots, J}\right\| \partial_{x_{m}} \rho_{k}(x, t) \|_{C_{b}\left(\mathbb{R}^{d}\right)} .
\end{aligned}
$$

(3) When $\mathcal{R}_{\ell}$ is of the type $S_{k}+S_{r} \rightarrow S_{j}+S_{i}$,

$$
\begin{aligned}
& \left|\partial_{x_{m}} \int_{\tilde{\boldsymbol{x}} \in \mathbb{X}^{(\ell)}} K_{\ell}(\tilde{\boldsymbol{x}})\left(\int_{\boldsymbol{y} \in \mathbb{Y}^{(\ell)}} \delta_{x}\left(y_{1}^{(j)}\right) m_{\ell}(\boldsymbol{y} \mid \tilde{\boldsymbol{x}}) d \boldsymbol{y}\right)\left(\Pi_{k=1}^{J} \Pi_{s=1}^{\alpha_{\ell k}} \rho_{k}\left(\tilde{x}_{s}^{(k)}, t\right)\right) d \tilde{\boldsymbol{x}}\right| \\
& =\left|\partial_{x_{m}} \int_{\mathbb{R}^{2 d}} K_{\ell}(z, w)\left(\int_{\mathbb{R}^{d}} m_{\ell}(x, y \mid z, w) d y\right) \rho_{k}(z, t) \rho_{r}(w, t) d z d w\right| \\
& \leq p \times\left|\partial_{x_{m}} \int_{\mathbb{R}^{d}} \hat{K}_{\ell}(x-y) \rho_{k}(x, t) \rho_{r}(y, t) d y\right|+(1-p) \times\left|\partial_{x_{m}} \int_{\mathbb{R}^{d}} \hat{K}_{\ell}(y-x) \rho_{k}(y, t) \rho_{r}(x, t) d y\right| \\
& =p \times\left|\int_{\mathbb{R}^{d}} \hat{K}_{\ell}(y) \partial_{x_{m}}\left(\rho_{k}(x, t) \rho_{r}(x-y, t)\right) d y\right|+(1-p) \times\left|\int_{\mathbb{R}^{d}} \hat{K}_{\ell}(-y) \partial_{x_{m}}\left(\rho_{k}(x-y, t) \rho_{r}(x, t)\right) d y\right| \\
& \leq k_{\ell} \times\left. 2 \sup _{k=1, \cdots, J}\left\|\partial_{x_{m}} \rho_{k}(x, t)\right\|_{C_{b}\left(\mathbb{R}^{d}\right)}\|\boldsymbol{\rho}\|\right|_{M} \leq 2 k_{\ell} C_{2} \sup _{k=1, \cdots, J}^{\left\|\partial_{x_{m}} \rho_{k}(x, t)\right\|_{C_{b}\left(\mathbb{R}^{d}\right)} .}
\end{aligned}
$$

(4) When $\mathcal{R}_{\ell}$ is of the type $S_{k} \rightarrow S_{j}+S_{r}$, without loss of generality, let us assume $\alpha_{i}>0$ for all $i=1, \cdots, I$. Then,

$$
\begin{aligned}
& \left|\partial_{x_{m}} \int_{\tilde{\boldsymbol{x}} \in \mathbb{X}^{(\ell)}} K_{\ell}(\tilde{\boldsymbol{x}})\left(\int_{\boldsymbol{y} \in \mathbb{Y}^{(\ell)}} \delta_{x}\left(y_{1}^{(j)}\right) m_{\ell}(\boldsymbol{y} \mid \tilde{\boldsymbol{x}}) d \boldsymbol{y}\right)\left(\Pi_{k=1}^{J} \Pi_{s=1}^{\alpha_{\ell k}} \rho_{k}\left(\tilde{x}_{s}^{(k)}, t\right)\right) d \tilde{\boldsymbol{x}}\right| \\
& =\left|\partial_{x_{m}} \int_{\mathbb{R}^{d}} K_{\ell}(z)\left(\int_{\mathbb{R}^{d}} m_{\ell}(x, y \mid z) d y\right) \rho_{k}(z, t) d z\right| \\
& =\left|\partial_{x_{m}} \int_{\mathbb{R}^{d}} K_{\ell}(z)\left(\int_{\mathbb{R}^{d}} \rho(|x-y|) \sum_{i=1}^{I} p_{i} \times \delta\left(z-\left(\alpha_{i} x+\left(1-\alpha_{i}\right) y\right)\right) d y\right) \rho_{k}(z, t) d z\right| \\
& =\left|\sum_{i=1}^{I} p_{i} k_{\ell} \times \partial_{x_{m}} \int_{\mathbb{R}^{d}} \rho(|x-y|) \rho_{k}\left(\alpha_{i} x+\left(1-\alpha_{i}\right) y, t\right) d y\right| \\
& =\left|\sum_{i=1}^{I} p_{i} k_{\ell} \times \int_{\mathbb{R}^{d}} \rho(|w|) \partial_{x_{m}} \rho_{k}\left(x-\left(1-\alpha_{i}\right) w, t\right) d w\right| \leq k_{\ell} \sup _{k=1, \cdots, J}\left\|\partial_{x_{m}} \rho_{k}(x, t)\right\| \|_{C_{b}\left(\mathbb{R}^{d}\right)} .
\end{aligned}
$$

This concludes the proof of the lemma.

\section{REFERENCES}

[AP95] Ambrosetti, Antonio, and Giovanni Prodi. A primer of nonlinear analysis. No. 34. Cambridge University Press, 1995. [CGV19] Caputo, M. Cristina, Thierry Goudon, and Alexis F. Vasseur. "Solutions of the 4-species quadratic reaction-diffusion system are bounded and $C^{\infty}$-smooth, in any space dimension." Analysis \& PDE 12.7 (2019): 1773-1804.

[CFF19] Crevat, Joachim, Grégory Faye, and Francis Filbet. "Rigorous Derivation of the Nonlocal Reaction-Diffusion FitzhughNagumo System." SIAM Journal on Mathematical Analysis 51.1 (2019): 346-373.

[D76a] M. Doi, Second quantization representation for classical many-particle system, J. Phys. A: Math. Gen. 9 (1976), no. 9, pp. $1465-1477$.

[D76b] M. Doi, Stochastic theory of diffusion-controlled reaction, J. Phys. A: Math. Gen. 9 (1976), no. 9, pp. 1479-1495.

[ECM07] Erban, Radek, Jonathan Chapman, and Philip Maini. "A practical guide to stochastic simulations of reaction-diffusion processes." arXiv preprint arXiv:0704.1908 (2007).

[E10] Evans, Lawrence C. Partial differential equations. Vol. 19. American Mathematical Soc., 2010.

[FMT20] Fellner, Klemens, Jeff Morgan, and Bao Quoc Tang. "Global classical solutions to quadratic systems with mass control in arbitrary dimensions." Annales de l'Institut Henri Poincaré C, Analyse non linéaire. Vol. 37. No. 2. Elsevier Masson, 2020.

[JG89] Furter, J., and Michael Grinfeld. "Local vs. non-local interactions in population dynamics." Journal of Mathematical Biology 27.1 (1989): 65-80.

[GB200] Gibson, M. A., and J. Bruck. "Efficient Exact Stochastic Simulation of Chemical Systems with Many Species and Many Channels." The Journal of Chemical Physics 104 (2000): 1876-1899.

[G00] Gillespie, Daniel T. "The chemical Langevin equation." The Journal of Chemical Physics 113.1 (2000): 297-306. 
[HGG07] Hesthaven, Jan S., Sigal Gottlieb, and David Gottlieb. Spectral methods for time-dependent problems. Vol. 21. Cambridge University Press, 2007.

[IMS20] Isaacson, Samuel A., Jingwei Ma, and Konstantinos Spiliopoulos. "Mean Field Limits of Particle-Based Stochastic Reaction-Diffusion Models." arXiv preprint arXiv:2003.11868 (2020).

[GB96] Gourley, S. A., and N. F. Britton. "A predator-prey reaction-diffusion system with nonlocal effects." Journal of Mathematical Biology 34.3 (1996): 297-333.

[I13] Isaacson, Samuel A. "A convergent reaction-diffusion master equation." The Journal of chemical physics 139.5 (2013): 054101.

[IZ18] Isaacson, Samuel A., and Ying Zhang. "An unstructured mesh convergent reaction-diffusion master equation for reversible reactions." Journal of Computational Physics 374 (2018): 954-983.

[K20] Kostré, M., C. Schütte, F. Noé, and M. J. del Razo. "Coupling particle-based reaction-diffusion simluations with reservoirs mediated by reaction-diffusion PDEs." arXiv preprint (2020): 2006.00003v1.

[LSU68] O.A. Ladyženskaja, V.A. Solonnikov and N.N. Ural'ceva. Linear and Quasi-linear equations of parabolic type, Transactions of Mahtematical Monographs, American Mathematical Society, Volume 23, (1968).

[LLN19] Lim, Tau Shean, Yulong Lu, and James Nolen. "Quantitative Propagation of Chaos in the bimolecular chemical reaction-diffusion model." arXiv preprint arXiv:1906.01051 (2019). APA

[MNKS09] J. Muñoz-García, Z. Neufeld, B. N. Kholodenko, and H. M. Sauro, Positional information generated by spatially distributed signaling cascades, PLoS Comp. Biol. 5 (2009), no. 3, e1000330.

[NTY17] Ninomiya, Hirokazu, Yoshitaro Tanaka, and Hiroko Yamamoto. "Reaction, diffusion and non-local interaction." Journal of Mathematical Biology 75.5 (2017): 1203-1233.

[NTS08] S. Neves, P. Tsokas, A. Sarkar, E. Grace, P. Rangamani, S. Taubenfeld, C. Alberini, J. Schaff, R. Blitzer, I. Moraru, and R. Iyengar, Cell shape and negative links in regulatory motifs together control spatial information flow in signaling networks, Cell 133 (2008), no. 4, pp. 666-680.

[PGB19] Pal, Swadesh, S. Ghorai, and Malay Banerjee. "Effect of kernels on spatio-temporal patterns of a non-local preypredator model." Mathematical biosciences 310 (2019): 96-107.

[P10] Pierre, Michel. "Global existence in reaction-diffusion systems with control of mass: a survey." Milan Journal of Mathematics 78.2 (2010): 417-455.

[S18] Souplet, Philippe. "Global existence for reaction-diffusion systems with dissipation of mass and quadratic growth." Journal of Evolution Equations 18.4 (2018): 1713-1720.

[SU17] Schneider, Guido, and Hannes Uecker. Nonlinear PDEs. Vol. 182. American Mathematical Soc., 2017.

[TS67] E Teramoto and N Shigesada, Theory of bimolecular reaction processes in liquids, Prog. Theor. Phys. 37 (1967), no. 1, pp. 29-51.

[SVB13] Segal, B. L., V. A. Volpert, and Alvin Bayliss. "Pattern formation in a model of competing populations with nonlocal interactions." Physica D: Nonlinear Phenomena 253 (2013): 12-22.

[SSS20] Shi, Qingyan, Junping Shi, and Yongli Song. "Effect of spatial average on the spatiotemporal pattern formation of reaction-diffusion systems." arXiv preprint arXiv:2001.11960 (2020).

[ZI20] Zhang, Y., L. Clemens, J. Goyette, J. Allard, O. Dushek, and S. A. Isaacson. "The Influence of Molecular Reach and Diffusivity on the Efficacy of Membrane-Confined Reactions." Biophysical J. 117 (2020): 1189-1201.

[BMP96] F. Baras, M. M. Mansour, and J. Pearson, Microscopic simulation of chemical bistability in homogeneous systems, The Journal of chemical physics, 105 (1996), pp. 8257-8261.

[BPM90] F. Baras, J. Pearson, and M. M. Mansour, Microscopic simulation of chemical oscillations in homogeneous systems, The Journal of chemical physics, 93 (1990), pp. 5747-5750.

[DYK18] A. Donev, C.-Y. Yang, and C. Kim, Efficient reactive brownian dynamics, The Journal of chemical physics, 148 (2018), p. 034103.

[KNBGD17] C. Kim, A. Nonaka, J. B. Bell, A. L. Garcia, and A. Donev, Stochastic simulation of reaction-diffusion systems: A fluctuating-hydrodynamics approach, The Journal of chemi- cal physics, 146 (2017), p. 124110. 